This is not the version of record. The full version of: Tetley, Robert J., Staddon, Michael F., Heller, Davide, Hoppe, Andreas, Banerjee, Shiladitya and Mao, Yanlan (2019) Tissue fluidity promotes epithelial wound healing. Nature Physics, 15(11), pp. 1195-1203 can be found at https://doi.org/10.1038/s41567-019-0618-1 


\section{Tissue Fluidity Promotes Epithelial Wound Healing}

3 Robert J. Tetley ${ }^{1,2}$, Michael F. Staddon ${ }^{2,3}$, Davide Heller ${ }^{4,5}$, Andreas Hoppe ${ }^{6}$,

4 Shiladitya Banerjee ${ }^{2,3}$, Yanlan $\mathrm{Mao}^{1,2,7 *}$

5 (1) MRC Laboratory for Molecular Cell Biology, University College London, Gower

6 Street, London WC1E 6BT, United Kingdom

7 (2) Institute for the Physics of Living Systems, University College London, London,

8 United Kingdom

9 (3) Department of Physics \& Astronomy, University College London, London, United

10 Kingdom

11 (4) Institute of Molecular Life Sciences, University of Zurich, Winterthurerstrasse 190,

12 Zurich, 8057, Switzerland

13 (5) SIB Swiss Institute of Bioinformatics, Quartier Sorge, Batiment Genopode,

14 Lausanne, 1015, Switzerland

15 (6) Faculty of Science, Engineering and Computing, Kingston University, Kingstonupon-Thames, United Kingdom

17 (7) College of Information and Control, Nanjing University of Information Science and

18 Technology, Nanjing, Jiangsu 210044, China

19 * Correspondence: y.mao@ucl.ac.uk 


\section{Summary}

23 The collective behaviour of cells in epithelial tissues is dependent on their

24 mechanical properties. However, the contribution of tissue mechanics to wound

25 healing in vivo remains poorly understood. Here we investigate the relationship

26 between tissue mechanics and wound healing in live Drosophila wing imaginal discs

27 and show that by tuning epithelial cell junctional tension, we can systematically alter

28 the rate of wound healing. Coincident with the contraction of an actomyosin purse string, we observe cells flowing past each other at the wound edge by intercalating, reminiscent of molecules in a fluid, resulting in seamless wound closure. Using a cell-based physical model, we predict that a reduction in junctional tension fluidises the tissue through an increase in intercalation rate and corresponding reduction in bulk viscosity, in the manner of an unjamming transition. The resultant fluidisation of

34 the tissue accelerates wound healing. Accordingly, when we experimentally reduce tissue tension in wing discs, intercalation rate increases and wounds repair in less time.

\section{Main Text}

Epithelial tissues are inevitably damaged from time to time and must therefore have robust repair mechanisms. The behaviour of cells and tissues, for instance

41 during wound healing and morphogenesis, depends on their mechanical properties

42 and those of the surrounding environment ${ }^{1}$. Previous studies have investigated the

43 contribution of tissue mechanics to epithelial wound healing primarily using in vitro

44 cell culture assays ${ }^{2-7}$. However, whether these in vitro assays truly recapitulate the

45 physiological response of in vivo tissues is unclear. Many in vivo models of epithelial 
46 wound healing have been developed ${ }^{7}$, although the mechanical basis of the wound

47 healing process in such in vivo systems remains unclear. A number of in vivo

epithelia have been shown experimentally to form contractile supracellular

actomyosin cables ${ }^{8-12}$ at the margin of epithelial wounds, which contract in a purse-

string manner and bring the edges of the wound into apposition. While the

mechanical role of the actomyosin purse string has begun to be investigated in

vivo $^{13,14}$, the contribution of surrounding tissue mechanical properties to wound healing remains unclear.

To investigate the role of tissue mechanics in wound healing in an in vivo system, we studied tissue repair in the epithelium of Drosophila wing imaginal discs by live time-lapse imaging. After wounding wing discs by laser ablation, an actomyosin purse string assembles at the wound's leading edge (Fig. 1a, Supplementary Video 1), as in other systems ${ }^{10,15-18}$. To understand the mechanisms controlling repair dynamics, we quantitatively analysed ${ }^{19}$ wound morphology over the time course of wound closure. We observe three distinct phases after wounding: recoil, fast closure and slow closure (Fig. 1b, Supplementary Video 2). Immediately after wounding, the wound area increases, due to a release of tissue tension by the ablation. The wound area then reduces in time, with an initial fast phase. However, after reaching approximately $50 \%$ of the original wound area, the rate of wound closure decreases dramatically, until closure. Myosin II (Myoll) intensity increases after wounding, before peaking at roughly twice the initial intensity (Fig. 1c). This peak coincides with the transition between fast and slow closure phases.

The dramatic reduction in wound closure rate at later stages suggested that different cell behaviours may be responsible for the fast and slow phases. By closely examining cell behaviours around the wound, we observe that cells at the wound 
71 edge readily undergo intercalation, particularly during the slow closure phase (Fig.

72 1d, e, g, Supplementary Video 2). This cell behaviour differs from those associated with wound healing in other Drosophila tissues, such as cell fusion ${ }^{20,21}$, polyploidisation $^{21}$ and cell intercalation further from the wound edge $\mathrm{e}^{22}$. We also saw no evidence of protrusion-based active migration or cell extrusion. During wound edge intercalations, junctions in contact with the wound shrink to a single vertex and new junctions grow in the orthogonal direction (Fig. 1d). As a result, the number of cells in contact with the wound decreases over time (Fig. 1e, Supplementary Fig. 1ad). These wound edge intercalations (involving three cells) are distinct from T1 transitions observed in many systems ${ }^{23}$, as a T1 transition involves the rearrangements of four neighbouring cells (see methods). The rate at which wound edge cells intercalate is roughly eleven times that of an unwounded tissue (Fig. 1f) and there is a strong relationship between wound edge intercalation and the reduction in wound area during the slow phase of wound closure (Fig. 1g), indicating that wound edge cell intercalation might be required to promote the completion of wound closure.

To quantitatively test the role of wound edge cell intercalation, we developed a computational vertex model ${ }^{24}$ for wound closure in the wing disc (Supplementary Fig. 2, see methods). While the mechanics of wound healing has been previously modelled using continuum ${ }^{2,4,6}$ or finite element approaches ${ }^{5,25}$, more representative cell-based models, such as vertex models ${ }^{26,27}$, have rarely been applied to replicate in vivo wound healing dynamics. We model each cell in the tissue as a twodimensional polygon carrying variable tension on their edges, with bulk elasticity and peripheral contractility (Supplementary Fig. 2a-b, methods). We parameterised the model so that edges contacting the wound gradually increase in tension compared to 
the surrounding tissue, to mimic the assembly of the contractile actomyosin purse string, causing wound edge junctions to reduce in length. To capture experimentally observed fluctuations in junctional and purse-string $\mathrm{Myoll}^{28}$, we introduced fluctuations in line tension at cell-cell interfaces and in the purse-string (Supplementary Fig. 2d). Without introducing intercalation events into the model, simulated wounds are unable to close (Figs. 2a, c, Supplementary Video 3). By contrast, when intercalations are enabled in the model (Supplementary Fig. 2c, see methods), wounds are able to close (Figs. 2b, d, Supplementary Video 4), supporting our hypothesis that intercalations at the wound edge are necessary to drive wound closure.

The vertex model predicts that in the absence of intercalation, cells around the wound become more elongated towards the centre of the wound (Fig. 2e) than in simulations with intercalations enabled (Fig. 2f). In both cases, the cells initially elongate as the purse-string contracts the wound. As cells begin to intercalate away from the wound edge their shapes relax, reducing the elongation over time. Towards the end of wound closure, many intercalations occur (Fig. 2b), at which point the elongation rapidly decreases, and the cells return to a fully relaxed state after healing (Fig. 2f). With intercalations disabled, the cells remain highly elongated (Fig. 2e). This led us to hypothesise that wound edge intercalations play a crucial role in maintaining cell shape and tissue patterning. Indeed, wing disc cells appear regularly packed immediately after wound closure (Fig. 3a) and the polygon distribution of wound edge cells is restored upon healing (Fig. 3b). The seamless closure we observe is distinct from a number of in vivo ${ }^{22,29}$ and in vitro ${ }^{5,30}$ systems that can form visible scar-like rosette structures upon closure. To test our vertex model's prediction that intercalation preserves cell shape, we quantified cell elongation in the first three 
121 rows of cells away from the wound in wing discs (Fig. 3c, Supplementary Video 5).

122 While cells in the second and third rows undergo little change in elongation during

123 wound closure (Fig. 3d), cells in the first row undergo a transient increase in

124 elongation towards the wound (Fig. 3c-f) before returning to their original shapes

125 prior to wound closure (Figs. 3d-f). Cells return to their original shape during the slow

126 phase of closure (Fig. 3d-e), when the majority of intercalations occur, supporting the

127 role of intercalation in preserving cell shape.

If intercalations are indeed required to maintain cell shape, we expect that the

majority of cell intercalations should be localised to the first row of cells, as it is these cells that are being stretched (Fig. 3d). Indeed, when we quantify the intercalation

rates in different rows of cells across the closure process, we find that the

intercalation rate is significantly higher in the first row of cells and decays in

increasing rows away from the wound edge (Fig. 3g, Supplementary Fig. 3 a-c). This

prediction of a localised increase in intercalation rate also holds true for our vertex

model (Fig. 3g, Supplementary Fig. 3b-c). However, when the strength of the purse

string is reduced the intercalation rate in the first row of cells is significantly lower

(Fig. 3g, Supplementary Fig. 3b-c). Therefore, the cell shape changes induced by

the close proximity of a cell (or row of cells) to the purse string can explain the

localised increase in intercalation rate that we observe. This mechanical function of

the purse string contrasts previous work suggesting that the purse-string functions

mainly in setting the direction of cell movement towards the wound during wound

142 closure ${ }^{5}$

Altogether, these findings suggested that intercalation events help maintain

cell shape and that wound healing in an epithelium that does not intercalate will lead 
146 in the wing disc to the Drosophila embryonic ectoderm, an epithelium in which cells

147 do not return to their original shape (Fig. 3h). Following wound closure in the

148 Drosophila embryonic ectoderm, cells can be up to twice as elongated as they were

149 prior to wounding ${ }^{22}$. Supporting the model's predictions, there are significantly fewer

150 wound edge intercalations in embryos prior to wound closure (Fig. 3i).

These results suggested that wound closure is controlled by two dynamic

mechanical properties of the wounded tissue: the rate of wound edge intercalation

and the tension in the purse string. We used the model to test the relative roles of

intercalation rate and purse string tension in wounded tissues. We found that the rate

of intercalation in the tissue can be tuned by modulating cell-cell interfacial tension

(Fig. 4a). Likewise, intercalation rate can also be tuned by modulating the perimeter

contractility of a cell (Supplementary Fig. 4a, b). As cell line tension decreases, the

rate of intercalation in the tissue increases. This leads to an increase in "tissue

fluidity" - the rearrangement of cells relative to each other, analogous to molecules in a liquid.

To demonstrate that a reduction in interfacial tension (increase in intercalations) indeed increases the fluidity of a tissue, we performed shear rheology simulations using our vertex model (Supplementary Fig. 5a), and computed the tissue shear modulus and viscosity with varying line tensions (Fig. 4b, Supplementary Fig. 5b-f). As line tension is gradually reduced in simulations, both the shear modulus and viscosity, the inverse of fluidity, approach zero ${ }^{31}$. Our findings stand in contrast to previous studies, which suggested that cell monolayers displaying viscous-like behaviours with many cell rearrangements ${ }^{32}$ fail to close wounds ${ }^{6}$. However, our results are consistent with the suggestion that increasing monolayer elasticity would prevent wound closure². 
Simulations using our vertex model also demonstrate that the intercalation

172 rate can increase rapidly when the magnitude of line tension fluctuations is increased

173 (Supplementary Fig. 4d), leading to a smaller corresponding reduction in closure

174 time (Supplementary Fig. 4c). However, when the tension fluctuations are large, the

175 intercalation rate in unwounded simulations far exceeds what we observe in

176 unwounded tissues (Fig. 1f) with many short timescale reversible intercalations. We

177 do not observe large numbers of reversible intercalations in the wing disc, therefore

178 it is unlikely that higher tension fluctuations are physiologically relevant. Simulations

179 also demonstrate that cell division rates can affect closure time (Supplementary Fig.

$1804 \mathrm{e})$. However, the range of cell division rates used in these simulations far exceeds

181 the physiological range of cell division rates we have measured in a number of

182 genetic conditions (Supplementary Fig. 4e). Therefore it is unlikely that cell division

183 has a strong contribution to wing disc wound closure for wounds of this size, unlike

184 for larger wounds ${ }^{33}$. Although varying the magnitude of tension fluctuations and

185 divisions rates is not physiologically relevant, the observation that both can be

186 modulated to increase fluidity, and subsequently wound closure rate (Supplementary

187 Fig. 4c-f), demonstrates that fluidity-driven wound closure is likely to be a general

188 phenomenon.

Increasing either purse string tension or decreasing cell line tension reduces the time taken for wounds to close (Fig. 4c) and increases the rate of wound edge intercalations (Fig. 4d). Unexpectedly, we find a region of parameter space where a reduction in purse string tension is more than compensated for by an increase in tissue fluidity (decrease in tension), leading to accelerated wound closure (Figs. 4c, e-f, magenta compared to black). However, tissue fluidity can only rescue a reduced 195 purse string up to a point, as the majority of simulations with the weakest purse 
196 string tension values fail to close, regardless of the level of tissue fluidity (Figs. 4c, e-

197 f). This observation highlights the importance of the purse string for the increase in wound edge intercalation (Fig. 3g, Supplementary Fig. 3). Together, these results demonstrate that bulk tissue mechanical properties and the activity of the purse string cooperate to promote wound closure.

To test these predictions from our vertex model further, we sought to experimentally perturb cell edge tension. In epithelial tissues, cell edge tension is governed by the activity of non-muscle Myoll (Fig. 5a). To test the roles of purse activity of Myoll in the wing pouch epithelium. To increase tension, we performed RNAi against the Myosin binding subunit (Mbs) of the Myosin Phosphatase, a phosphatase that inactivates Myoll by dephosphorylating its regulatory light chain ${ }^{34}$ (Spaghetti squash (Sqh) in Drosophila). To decrease tension, we performed RNAi against Rho-kinase (Rok), a kinase that activates Myoll by phosphorylating Sqh ${ }^{35,36}$. We confirmed the effect of these genetic perturbations on tension by quantifying vertex recoil rates (a greater recoil rate implying higher tension) after single junction ablations in unwounded tissues (Fig. $5 \mathrm{~g}$ ). We then wounded these wing discs and compared the dynamics of wound closure to wildtype (WT) wing discs (Supplementary Fig. 6a-d). In Mbs RNAi wing discs, where tension is high, wounds fail to close within the imaging time window (Figs. 5b, c, Supplementary Fig. 6a, Supplementary Fig. 7, Supplementary Video 6). Furthermore, wound edge cell intercalation is almost entirely abolished (Figs. 5c, e-f, Supplementary Fig. 7, Supplementary Video 6), supporting the importance of intercalation in promoting wound closure. 

which tension is reduced, wounds close faster than in WT wing discs. While these wounds initially close more slowly than WT wounds, they eventually overtake them and unexpectedly close in roughly half the time of WT wounds (Figs. 5b, d,

224 Supplementary Fig. 6a). This is accompanied by an increase in the rate of wound edge intercalation (Figs. 5d-f). A decrease in interfacial tension can also be achieved via an increase in cell-cell adhesion. An increase in cell-cell adhesion is thought to promote cell-cell contact formation, effectively counteracting junction contractility ${ }^{37}$. In line with this prediction, we obtained intercalation and closure rates similar to Rok RNAi wing discs when we expressed double the number of copies of the adherens junction-associated cell-cell adhesion molecule E-cadherin (ubi-Ecad-GFP, Supplementary Fig. 6a-d, Supplementary Fig. 9a-h). Therefore, decreased interfacial tension appears to be a general route to increasing tissue fluidity and wound closure rate, regardless of the origin of the tension decrease.

One consequence of decreasing Myoll activity through Rok RNAi, is that it is likely to increase tissue fluidity, while simultaneously decreasing purse string tension $^{8,38}$. To quantify the effects of Myoll perturbations on tension in the surrounding tissue and in the purse string, we ablated single junctions at the wound edge and in the surrounding tissue in all three conditions and compared the initial vertex recoil velocities that serve as a measure of junctional tension ${ }^{39}$ (Fig. $5 \mathrm{~g}$, Supplementary Table 1). In WT tissues, the junction recoil rate in the purse string is twice that of the surrounding tissue. This mimics the relative increase in Myoll intensity we observed in WT wing discs (Fig. 1c). In Rok RNAi wing discs, vertex recoil rates are reduced by $51 \%$ in the tissue and $35 \%$ in the purse string compared to WT. One would therefore predict that the increase in tissue fluidity in Rok RNAi 
245 discs, associated with a large decrease in tension in the surrounding tissue, may be 246 able to more than compensate for the reduction in purse string strength, as predicted 247 by our model (Fig. 4c). In Mbs RNAi wing discs, vertex recoil rates are increased by $24840 \%$ in the tissue and decreased by $21 \%$ in the purse string compared to WT. The 249 decrease in both tissue fluidity (due to increased tissue junctional tension) and purse 250 string strength likely explains why wounds in Mbs RNAi discs fail to close.

251 Interestingly, Mbs RNAi purse string vertex recoil rates are not significantly higher 252 than vertex recoil rates in either the WT purse string or Mbs RNAi tissue (Fig. $5 g$, 253 Supplementary Table 1). This suggests that we have elevated tension in the wing 254 disc to its maximum possible level through Mbs RNAi, a level that is also achieved in the purse string of WT discs. To test whether measured vertex recoil rates in the three experimental conditions can predict wound closure rates, we used the measured vertex recoil rates as an input to our model by assuming that the total edge tension is proportional to the recoil rate (Fig. 5h, see methods). We also used experimentally measured division rates for WT, Rok RNAi, and Mbs RNAi (Supplementary Fig. 4e), although these rates show little variation and cannot explain changes in wound closure rate. We find that the differences in experimentally measured tensions are sufficient to explain the increased healing rate in Rok RNAi and failed healing in Mbs RNAi (Fig. 5i).

We have thus demonstrated that wing disc wound closure is dependent on cell-cell intercalation-driven tissue fluidity, downstream of interfacial junctional tension. The dynamics of wound closure in wing discs can be explained purely through junctional behaviours in our vertex model, rather than previously described cell-crawling based migration ${ }^{30,40-42}$. In our system, fluidity may substitute for the propulsive action provided by cell crawling, as it provides a mechanism for energy 
270 dissipation, which leads to mechanical relaxation ${ }^{27}$ (Supplementary Fig. 10a-c). The

271 dispensability of crawling for our system is reminiscent of the closure of cell

272 monolayer wounds over non-adherent environments ${ }^{3,6}$, which entirely depends on

273 purse string tensile activity. In these studies, wounds close much slower than in wing

274 discs and a reinforcement of purse string strength is thought to be required for

275 closure $^{6}$. Both these differences may be explained by a failure of these monolayers

276 to efficiently dissipate energy, unlike in wing discs (Supplementary Fig. 10a-c).

277 Changes in tension-based fluidity, such as those we have induced through

278 Myoll perturbation, can be interpreted as an unjamming transition ${ }^{43,44}$. Epithelial

279 tissues can transition between a fluid-like state (unjammed) with many

280 rearrangements and a solid-like jammed state, lacking the ability to rearrange ${ }^{43-45}$.

281 Theoretical models have described how jamming/unjamming transitions can be

282 induced via changes in junctional tension and cell-cell adhesion ${ }^{46}$. These two

283 properties define a mechanical energy barrier, which must be overcome for cells to

284 rearrange relative to each other ${ }^{43,46}$, allowing an epithelium to behave as a fluid. By

285 reducing tension in the wing disc, we are likely lowering this energy barrier, allowing

286 cells to rearrange more. Simulations indeed demonstrate that, with increased

287 intercalations, tissues can transit to a lower energy state during wound healing,

288 which may allow further intercalations to occur (Supplementary Fig. 10a-c). The role

289 we describe for tissue fluidity in wound healing, in addition to its known roles in

290 developing ${ }^{28,47-50}$ and mature tissues ${ }^{46}$, reinforces the physiological importance of the

291 fluid state of a tissue.

References 


\begin{tabular}{|c|c|c|}
\hline 294 & 1 & Ladoux, B. \& Mege, R. M. Mechanobiology of collective cell behaviours. Nat Rev Mol Cell Bio \\
\hline 6 & 2 & Cochet-Escartin O. Ranft, J. Silberzan, P. \& Marca. P. Border Forces and Friction Control \\
\hline 97 & & Epithelial Closure Dynamics. Biophys J 106, 65-73, doi:10.1016/j.bpj.2013.11.015 (2014). \\
\hline $\begin{array}{l}98 \\
99\end{array}$ & 3 & $\begin{array}{l}\text { Nier, V. et al. Tissue fusion over nonadhering surfaces. P Natl Acad Sci USA 112, 9546-9551, } \\
\text { doi:10.1073/pnas.1501278112 (2015). }\end{array}$ \\
\hline 00 & 4 & Arciero, J. C., Mi, Q., Branca, M. F., Hackam, D. J. \& Swigon, D. Continuum Model of \\
\hline 01 & & $\begin{array}{l}\text { Collective Cell Migration in Wound Healing and Colony Expansion. Biophys J 100, 535-543, } \\
\text { doi:10.1016/j.bpj.2010.11.083 (2011). }\end{array}$ \\
\hline 03 & 5 & Brugues, A. et al. Forces driving epithelial wound healing. Mol Biol Cell 25 (2014). \\
\hline 304 & 6 & Vedula, S. R. K. et al. Mechanics of epithelial closure over non-adherent environments. Nat \\
\hline 05 & & Commun 6, doi:ARTN 6111 \\
\hline 306 & \multicolumn{2}{|c|}{ 10.1038/ncomms7111 (2015). } \\
\hline 307 & 7 & Begnaud, S., Chen, T. C., Delacour, D., Mege, R. M. \& Ladoux, B. Mechanics of epithelial \\
\hline 308 & & tissues during gap closure. Curr Opin Cell Biol 42, 52-62, doi:10.1016/j.ceb.2016.04.006 \\
\hline 309 & & (2016). \\
\hline 310 & 8 & Russo, J. M. et al. Distinct temporal-spatial roles for rho kinase and myosin light chain kinase \\
\hline 311 & & in epithelial purse-string wound closure. Gastroenterology 128, 987-1001, \\
\hline 312 & & doi:10.1053/j.gastro.2005.01.004 (2005). \\
\hline 313 & 9 & Abreu-Blanco, M. T., Verboon, J. M., Liu, R., Watts, J. J. \& Parkhurst, S. M. Drosophila \\
\hline 314 & & embryos close epithelial wounds using a combination of cellular protrusions and an \\
\hline 315 & & actomyosin purse string. J Cell Sci 125, 5984-5997, doi:10.1242/jcs.109066 (2012). \\
\hline 316 & 10 & Wood, W. et al. Wound healing recapitulates morphogenesis in Drosophila embryos. Nat \\
\hline 317 & & Cell Biol 4, 907-912, doi:10.1038/ncb875 (2002). \\
\hline 318 & 11 & Brock, J., Midwinter, K., Lewis, J. \& Martin, P. Healing of incisional wounds in the embryonic \\
\hline 319 & & chick wing bud: Characterization of the actin purse-string and demonstration of a \\
\hline 320 & & requirement for Rho activation. J Cell Biol 135, 1097-1107, doi:DOI 10.1083/jcb.135.4.1097 \\
\hline 321 & & (1996). \\
\hline 322 & 12 & Davidson, L. A., Ezin, A. M. \& Keller, R. Embryonic wound healing by apical contraction and \\
\hline 323 & & ingression in Xenopus laevis. Cell Motil Cytoskel 53, 163-176, doi:10.1002/cm.10070 (2002). \\
\hline 324 & 13 & Zulueta-Coarasa, T. \& Fernandez-Gonzalez, R. Dynamic force patterns promote collective cell \\
\hline 325 & & movements during embryonic wound repair. Nat Phys 14, 750-+, doi:10.1038/s41567-018- \\
\hline 326 & & 0111-2 (2018). \\
\hline 327 & 14 & Kobb, A. B., Zulueta-Coarasa, T. \& Fernandez-Gonzalez, R. Tension regulates myosin \\
\hline 328 & & dynamics during Drosophila embryonic wound repair. J Cell Sci 130, 689-696, \\
\hline 329 & & doi:10.1242/jcs.196139 (2017). \\
\hline 330 & 15 & Bement, W. M., Forscher, P. \& Mooseker, M. S. A Novel Cytoskeletal Structure Involved in \\
\hline 331 & & Purse String Wound Closure and Cell Polarity Maintenance. J Cell Biol 121, 565-578, doi:DOI \\
\hline 332 & & 10.1083/jcb.121.3.565 (1993). \\
\hline 333 & 16 & Martin, P. \& Lewis, J. Actin Cables and Epidermal Movement in Embryonic Wound-Healing. \\
\hline 334 & & Nature 360, 179-183, doi:DOI 10.1038/360179a0 (1992). \\
\hline 335 & 17 & Bement, W. M., Mandato, C. A. \& Kirsch, M. N. Wound-induced assembly and closure of an \\
\hline 336 & & actomyosin purse string in Xenopus oocytes. Curr Biol 9, 579-587, doi:Doi 10.1016/S0960- \\
\hline 337 & & 9822(99)80261-9 (1999). \\
\hline 338 & 18 & Danjo, Y. \& Gipson, I. K. Actin 'purse string' filaments are anchored by E-cadherin-mediated \\
\hline 339 & & adherens junctions at the leading edge of the epithelial wound, providing coordinated cell \\
\hline 340 & & movement. J Cell Sci 111, 3323-3332 (1998). \\
\hline 341 & 19 & Heller, D. et al. EpiTools: An Open-Source Image Analysis Toolkit for Quantifying Epithelial \\
\hline 342 & & Growth Dynamics. Dev Cell 36, 103-116, doi:10.1016/j.devcel.2015.12.012 (2016). \\
\hline 343 & 20 & Galko, M. J. \& Krasnow, M. A. Cellular and genetic analysis of wound healing in Drosophila \\
\hline 344 & & larvae. Plos Biol 2, 1114-1126, doi:ARTN e239 \\
\hline
\end{tabular}


10.1371/journal.pbio.0020239 (2004).

21 Losick, V. P., Fox, D. T. \& Spradling, A. C. Polyploidization and Cell Fusion Contribute to Wound Healing in the Adult Drosophila Epithelium. Curr Biol 23, 2224-2232, doi:10.1016/j.cub.2013.09.029 (2013).

22 Razzell, W., Wood, W. \& Martin, P. Recapitulation of morphogenetic cell shape changes enables wound re-epithelialisation. Development 141, 1814-1820, doi:10.1242/dev.107045 (2014).

23 Tetley, R. J. \& Mao, Y. The same but different: cell intercalation as a driver of tissue deformation and fluidity. Philosophical Transactions of the Royal Society B: Biological Sciences 373, doi:10.1098/rstb.2017.0328 (2018).

24 Fletcher, A. G., Osterfield, M., Baker, R. E. \& Shvartsman, S. Y. Vertex Models of Epithelial Morphogenesis. Biophys J 106, 2291-2304, doi:10.1016/j.bpj.2013.11.4498 (2014).

25 Wyczalkowski, M. A., Varner, V. D. \& Taber, L. A. Computational and experimental study of the mechanics of embryonic wound healing. J Mech Behav Biomed 28, 125-146, doi:10.1016/j.jmbbm.2013.07.018 (2013).

26 Barton, D. L., Henkes, S., Weijer, C. J. \& Sknepnek, R. Active Vertex Model for cell-resolution description of epithelial tissue mechanics. PLoS Comput Biol 13, e1005569, doi:10.1371/journal.pcbi.1005569 (2017).

27 Staddon, M. F. et al. Cooperation of dual modes of cell motility promotes epithelial stress relaxation to accelerate wound healing. PLoS Comput Biol 14, e1006502, doi:10.1371/journal.pcbi.1006502 (2018).

28 Curran, S. et al. Myosin II Controls Junction Fluctuations to Guide Epithelial Tissue Ordering. Dev Cell 43, 480-492 e486, doi:10.1016/j.devcel.2017.09.018 (2017).

29 Shindo, A. et al. Septin-dependent remodeling of cortical microtubule drives cell reshaping during epithelial wound healing. J Cell Sci 131, doi:UNSP jcs212647

10.1242/jcs.212647 (2018).

30 Anon, E. et al. Cell crawling mediates collective cell migration to close undamaged epithelial gaps. P Natl Acad Sci USA 109, 10891-10896, doi:10.1073/pnas.1117814109 (2012).

31 Bi, D. P., Lopez, J. H., Schwarz, J. M. \& Manning, M. L. A density-independent rigidity transition in biological tissues. Nat Phys 11, 1074-+, doi:10.1038/Nphys3471 (2015).

32 Vedula, S. R. et al. Epithelial bridges maintain tissue integrity during collective cell migration. Nat Mater 13, 87-96, doi:10.1038/nmat3814 (2014).

33 Bergantinos, C., Corominas, M. \& Serras, F. Cell death-induced regeneration in wing imaginal discs requires JNK signalling. Development 137, 1169-1179, doi:10.1242/dev.045559 (2010).

34 Vereshchagina, N. et al. The essential role of PP1 beta in Drosophila is to regulate nonmuscle myosin. Mol Biol Cell 15, 4395-4405, doi:10.1091/mbc.E04-02-0139 (2004).

35 Amano, M. et al. Phosphorylation and activation of myosin by Rho-associated kinase (Rhokinase). J Biol Chem 271, 20246-20249, doi:DOI 10.1074/jbc.271.34.20246 (1996).

36 Mizuno, T., Amano, M., Kaibuchi, K. \& Nishida, Y. Identification and characterization of Drosophila homolog of Rho-kinase. Gene 238, 437-444, doi:Doi 10.1016/S03781119(99)00351-0 (1999).

37 Lecuit, T. \& Lenne, P. F. Cell surface mechanics and the control of cell shape, tissue patterns and morphogenesis. Nat Rev Mol Cell Bio 8, 633-644, doi:10.1038/nrm2222 (2007).

38 Verboon, J. M. \& Parkhurst, S. M. Rho family GTPase functions in Drosophila epithelial wound repair. Small GTPases 6, 28-35, doi:10.4161/21541248.2014.982415 (2015).

39 Farhadifar, R., Roper, J. C., Algouy, B., Eaton, S. \& Julicher, F. The influence of cell mechanics, cell-cell interactions, and proliferation on epithelial packing. Current Biology 17, 2095-2104, doi:10.1016/j.cub.2007.11.049 (2007).

40 Chepizhko, O. et al. From jamming to collective cell migration through a boundary induced transition. Soft Matter 14, 3774-3782, doi:10.1039/c8sm00128f (2018). 


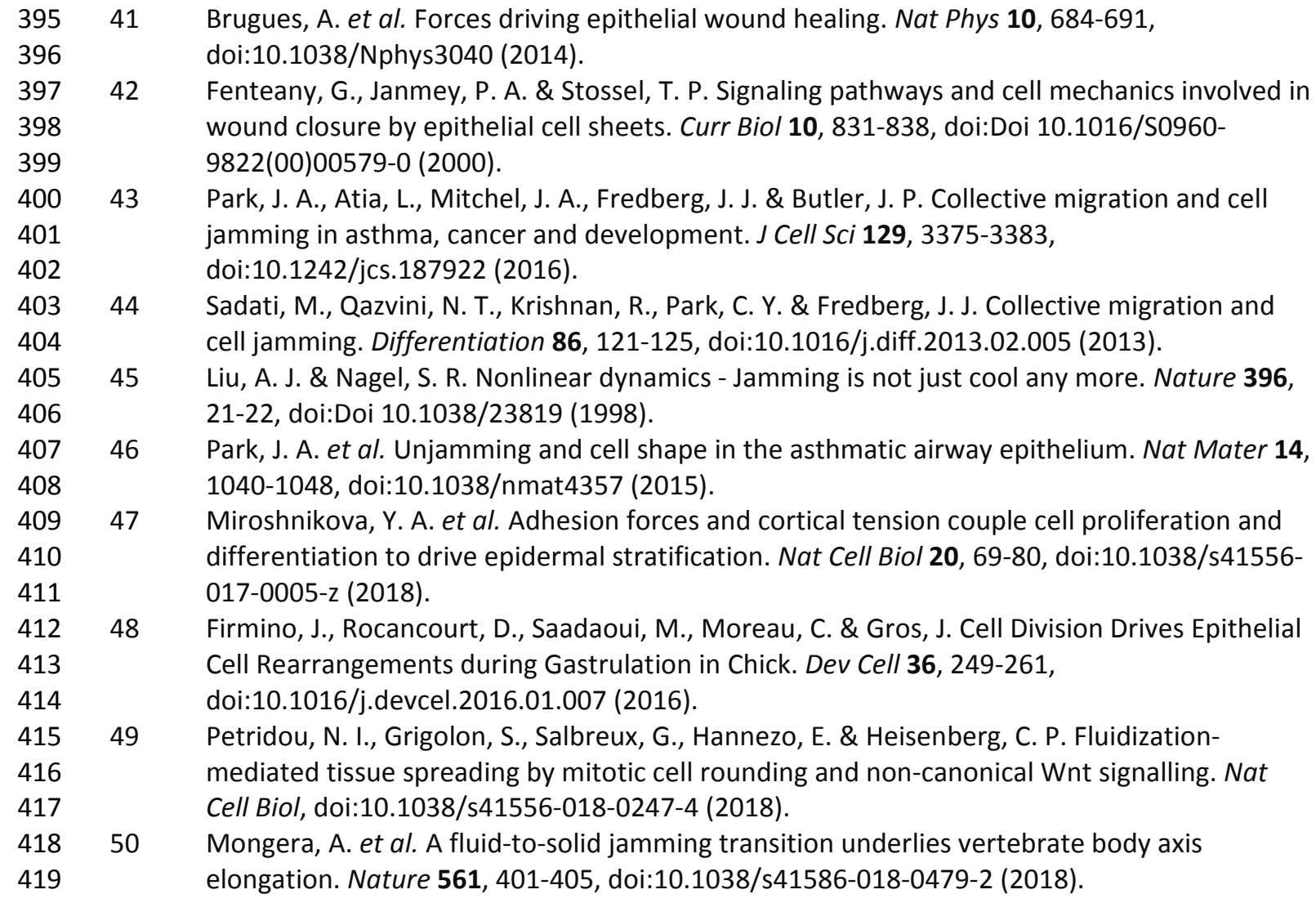

422 Thank you to all members of the Mao group, Martin Raff, David Ish-Horowicz and

423 Michael Murrell for providing feedback on the manuscript and thank you to the Baum 424 and Tapon labs for sharing fly stocks.

\section{Author Contributions}

427 RJT and YM conceived the experiments. SB and MFS conceived the theory. RJT 428 performed the experiments and analysed the data. MFS ran simulations and 429 analysed the data. $\mathrm{DH}$ and $\mathrm{AH}$ developed new image analysis tools in EpiTools and 430 wrote the corresponding methods. RJT, MFS, SB and YM wrote the manuscript. 
431

432 


\section{Author Information}

434 RJT was funded by a Medical Research Council Skills Development Fellowship 435 (MR/N014529/1). MFS is supported by an EPSRC funded PhD Studentship at the 436 UCL Department of Physics and Astronomy. DH was supported by the Swiss

437 National Science Foundation (31003A-160095). SB acknowledges support from 438 Royal Society University Research Fellowship (URFIR1 180187), and a Strategic 439 Fellowship from the UCL Institute for the Physics of Living Systems. YM is funded by 440 a Medical Research Council Fellowship (MR/L009056/1), a UCL Excellence 441 Fellowship, a NSFC International Young Scientist Fellowship (31650110472) and a 442 Lister Institute Research Prize Fellowship. This work was also supported by MRC 443 funding to the MRC LMCB University Unit at UCL (award code MC_U12266B).

\section{Competing Interests}

446 We confirm that the authors have no competing interests. 
Figure 1. Wing disc wound closure is punctuated by wound edge intercalation, which can drive wound closure

a, Early stages of wound closure in a $s q h^{A X 3} ;$ sqh-GFP, Ecad-tdTomato wing imaginal disc.

454 Cell outlines are marked by Ecad-tdTomato (green) and Myosin II by Sqh-GFP (magenta).

Cells which will be ablated are marked by white circles at 0 mins. Within the first 10 minutes after wounding, a strong accumulation of Myosin II can be seen at the wound's edge in the 
474 orthogonal direction. Scale bar $=3 \mu \mathrm{m}$. e, Quantification of the percentage of starting wound

475 edge junctions (magenta) and wound percentage area (green) for a single Ecad-GFP wing

476 disc wound. The percentage of junctions remaining on the wound's edge reduces as

477 intercalations occur until the wound fully closes. f, Quantification of intercalation rate in

478 unwounded WT tissues and at WT wound edges. The intercalation rate is significantly higher

479 at the wound edge (unpaired $t$-test with Welch's correction, $n=5, t=15.15, d f=5.089$,

$480 p<0.0001)$. Error bars $=$ SD. g, Relationship between percentage of wound start area and

481 percentage of starting wound edge junctions remaining. The mean percentage area at which

482 there is a transition between the fast (green) and slow (magenta) closure phases is

483 highlighted (57.6\%). A moving average curve ( \pm 4 time points) of the data is shown.

Figure 2. A vertex model of wound healing predicts that intercalation is necessary for wound closure and cell shape maintenance

a-f, Vertex model simulations. $\mathbf{a}, \mathbf{b}$, Percentage of initial wound area and wound junctions after ablation with intercalations (a) disabled, and (b) enabled. c, d, Vertex model simulation images after ablation with intercalations (c) disabled, and (d) enabled. e, f, Percentage change in cell elongation over time during and after wound closure, calculated by dividing the major axis by the minor axis of an ellipse fit to each cell, for the first three rows of cells around the wound with intercalations (e) disabled, and (f) enabled.

Figure 3. Wound edge intercalation preserves cell shape

a, Maximum intensity projection images of a wound in a $s q h^{A X 3}$; sqh-GFP, Ecad-tdTomato wing disc before (left) and immediately after (right) wound closure (scale bar $=3 \mu \mathrm{m}) . \mathbf{b}$, 
499 Quantification of wound edge cell polygon number before ablation, immediately after ablation 500 and immediately after wound closure. The distribution of polygon number is significantly 501 shifted left after ablation (Kolmogorov-Smirnov Test, $D=0.2892, p=0.0019$ ) but is restored 502 upon closure (Kolmogorov-Smirnov Test, $D=0.07583, p=0.9692)$. c, Colour coding of the first three rows away from the wound edge (first row blue, second row orange, third row yellow) mean cell elongation for the first three rows of cells (colour coding as in c) over time for 5 WT wounds. Moving average curves ( \pm 4 time points) are shown. The transition between fast and slow closure phases is marked by a dotted line (18.37 mins). e, Percentage change in mean elongation of first row cells for three time windows; 0-10 mins (wound recoil), 10-140 mins (early wound closure), 140 mins - close (late wound closure). Data is pooled from the data set in d. Cells were significantly elongated during the recoil phase (Wilcoxon Signed Rank Test, $p<0.0001)$. Cells were significantly more elongated during early wound closure than during late wound closure (Kolomogorov-Smirnov Test, $D=0.5703, p<0.0001$ ). Error bars = SD. $\mathbf{f}, \mathbf{h}$, Skeletonised cell outlines of cells starting at the wound edge (wound shaded discs $(n=5)$ (unpaired $t$-test with Welch's correction, $t=5.466, d f=2.103, p=0.0285$ ). 
527 Figure 4. Reducing tissue contractility enhances fluidity and can speed wound

528 closure

529

530 All figures are vertex model simulations. a, Intercalation rate in an unwounded tissue against

531 mean cell line tension. Error bars $=$ SD. $\mathbf{b}$, Normalized tissue shear modulus and viscosity

532 against tension. c, Mean wound closure time against cell line tension and purse-string

533 tension. The white region indicates parameter space where wounds fail to close within 300

534 minutes. The colored stars indicate the parameters used in (e) and (f). d, Mean wound edge

535 intercalation rate against cell line tension and purse-string tension. e, Percentage of initial

536 area over time for low, moderate, and high line tension cases. Points are from simulations,

537 and lines are fit dual exponential curves. f, Percentage of initial wound junctions over time

538 for low, moderate, and high tension cases. Points are from simulations; lines are the average

539 over all simulations. For all combinations of tension, $\mathrm{n}=12$ simulations for each.

Figure 5. Myosin activity controls tissue fluidity and wound closure rate

a, Activation of Myosin II by phosphorylation of its regulatory light chain (Sqh) can be performed by Rho kinase (Rok) downstream of Rho1. Myosin II inactivation by Sqh dephosphorylation can be performed by the Myosin Phosphatase comprising the Myosin binding subunit (Mbs) and catalytic subunit (Flapwing, Flw). b, Quantification of wound closure (as percentage of start wound area) over time in Mbs RNAi (green, n=5) and Rok RNAi (magenta, $n=5$ ) wing discs, compared to WT wound closure (black, $n=5$ ). Two-phase exponential decays are fitted after 3 minutes. c, d, Examples of wound healing in (c) Mbs 
551 RNAi and (d) Rok RNAi before wounding (left) and after wound closure (right, Rok RNAi) or 552 when further segmentation become impossible (right, Mbs RNAi). Cells are colour coded 553 according to whether they undergo intercalation (dark blue) or not (cyan). Images are 554 adaptive projections of Ecad-GFP overlaid by skeletonised cell outlines in cyan (scale bars = $5553 \mu \mathrm{m}) . \mathbf{e}$, Quantification of the percentage of initial wound edge junctions over time for Mbs 556 RNAi and Rok RNAi wing discs (colours and $n$ numbers as in b). Moving average curves ( \pm 4 557 time points) are shown. f, Quantification of mean intercalation rate for Mbs RNAi, WT and 558 Rok RNAi wounds (colours and n numbers as in b). Intercalation rate is significantly higher in 559 Rok RNAi wounds (unpaired $t$-test, $\mathrm{n}=5, t=8.026, d f=8, p<0.0001$ ) and significantly lower in 560 Mbs RNAi wounds (unpaired $t$-test, $\mathrm{n}=5, t=8.503, d f=8, p<0.0001$ ) compared to WT. Error 561 bars $=$ SD. g, Quantification of initial vertex recoil rates after single junction ablations in WT 562 (black), Mbs RNAi (green) and Rok RNAi (magenta) wing discs. Junction ablations were 563 performed in unwounded tissues ("Tissue") and at wound edges ("Wound"). Wound edge 564 vertex recoil rates were significantly higher than in the surrounding tissue in WT and Rok 565 RNAi discs, but not Mbs RNAi discs. Vertex recoil rates at the wound edge were significantly 566 lower in Rok RNAi discs compared to WT discs, but were not significantly changed in Mbs 567 RNAi discs. Full results of Kolmogorov Smirnov tests can be found in Supplementary Table 568 1. h, Input mean and standard deviation of effective tension, the total of line tension and 569 contractility, for different simulated conditions. The left bars are for bulk edges, and right 570 edges are for wound edges. i, Mean wound closure time for the simulated conditions. Mbs

571 RNAi simulated wounds fail to heal. Error bars = SD. Wound closure time is significantly 572 lower in Rok RNAi wounds than WT wounds (unpaired $t$-test, $\mathrm{n}=10, t=6.298, d f=18$, $573 p<0.0001)$. 
a Ecad-tdTomato Sqh-GFP
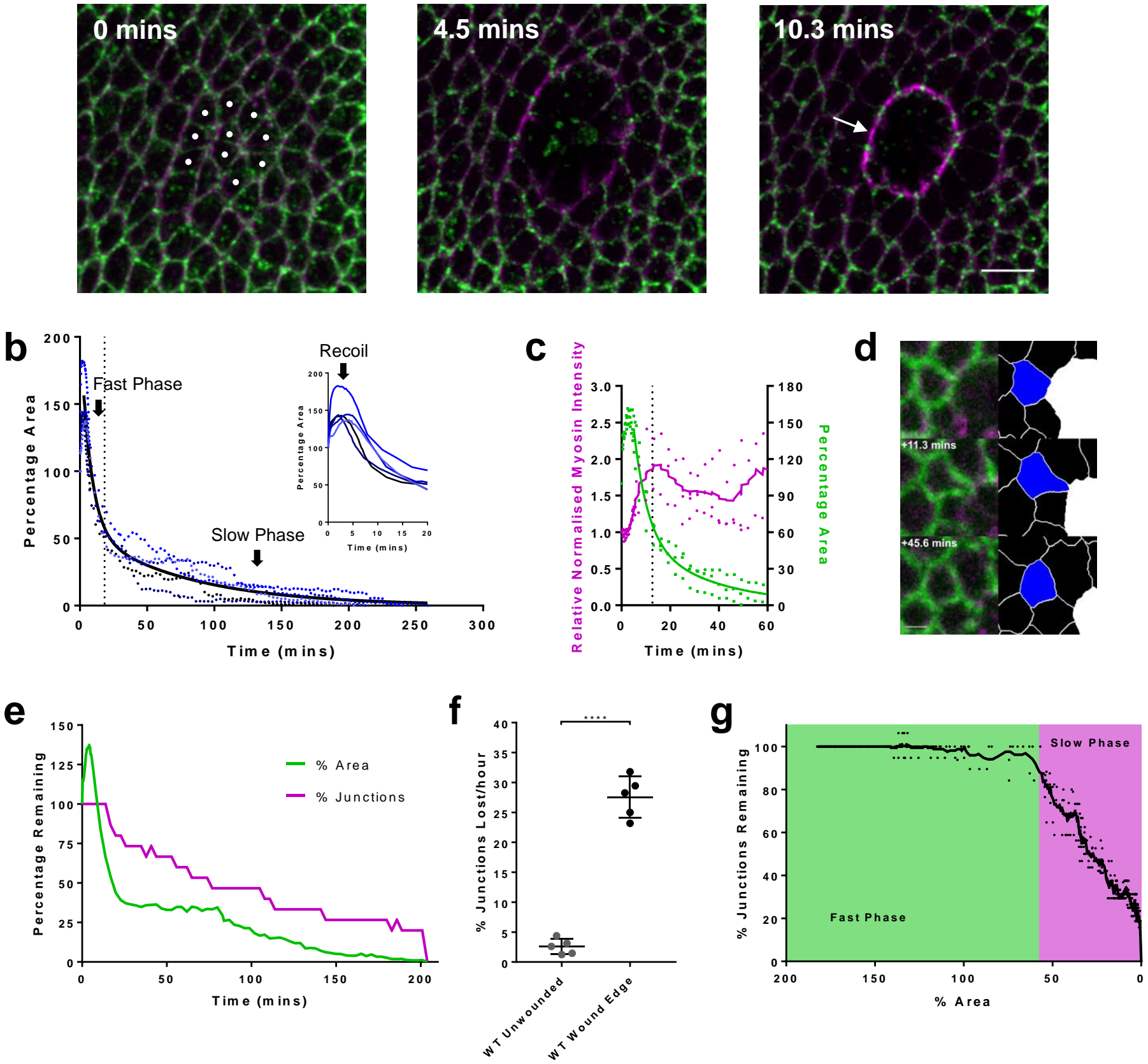

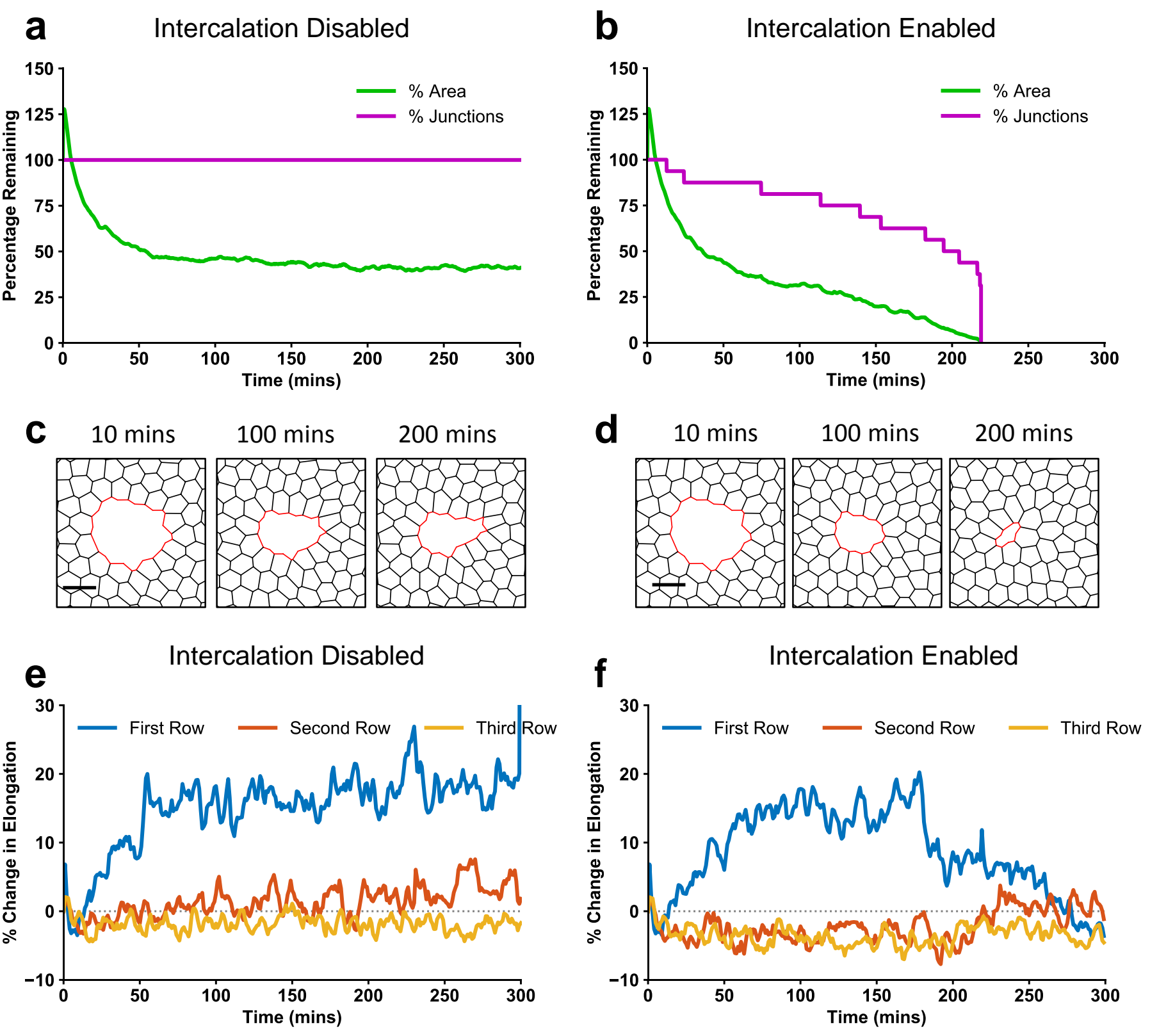
a Ecad-tdTomato Sqh-GFP
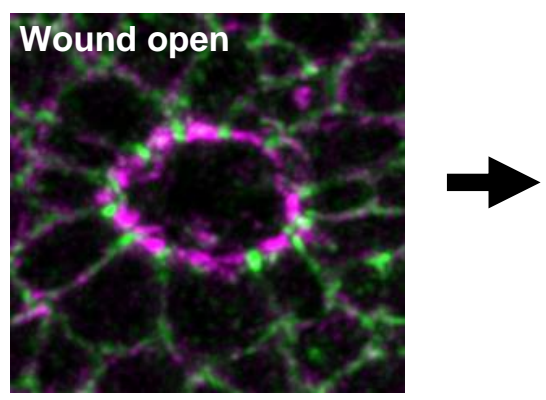

C

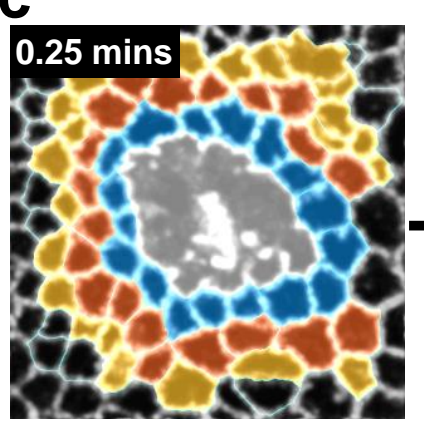

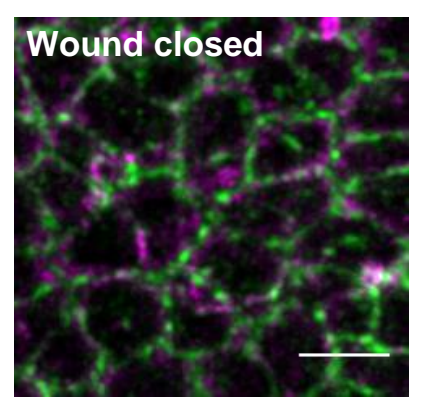

d

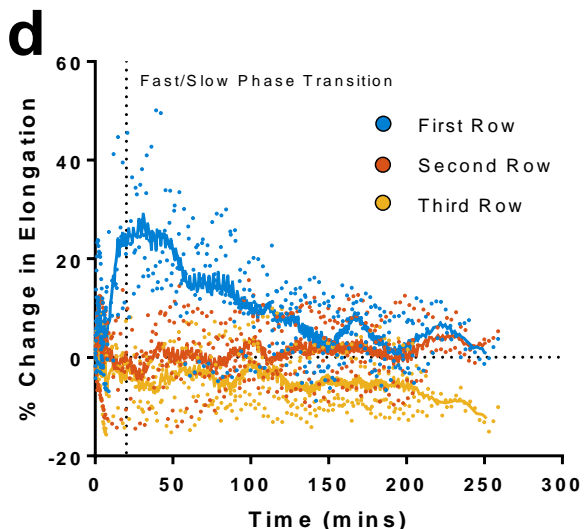

b

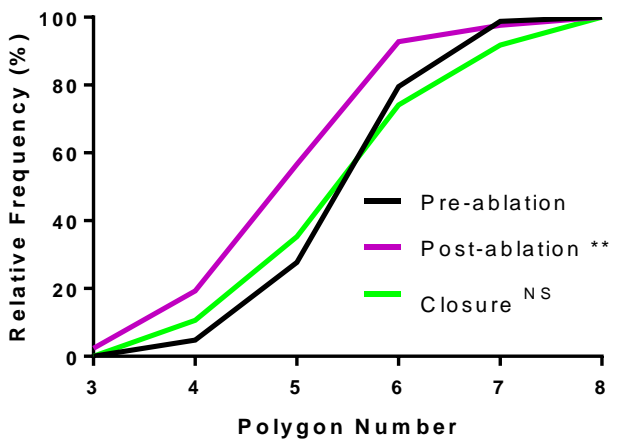

e

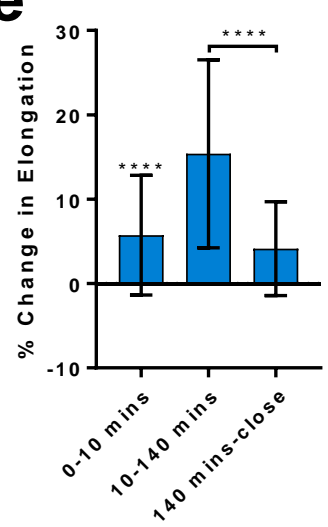

f Wing Disc 0 mins

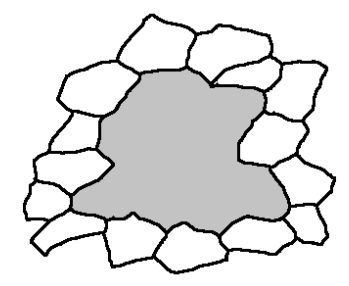

h Embryo

0 mins

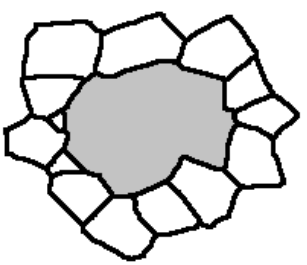

29 mins

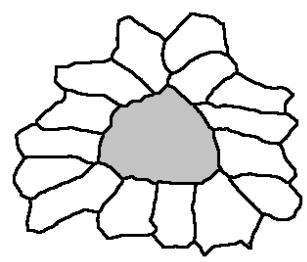

37 mins

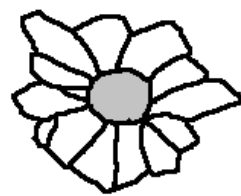

204 mins

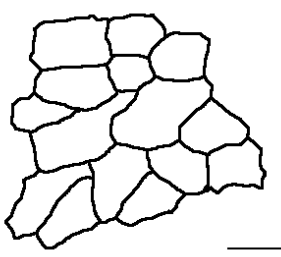

74 mins

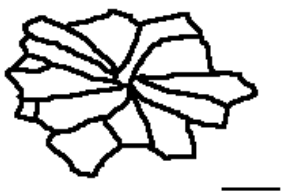

g

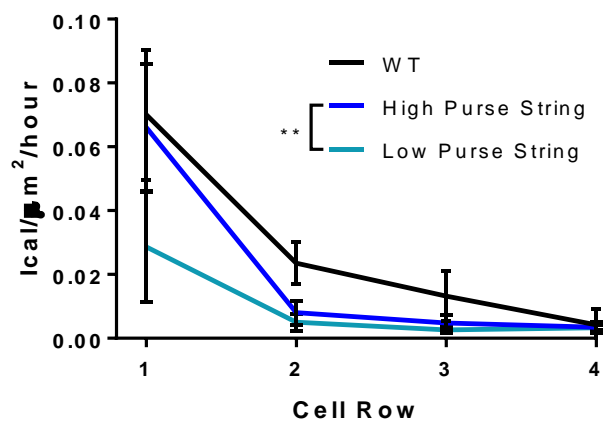

i

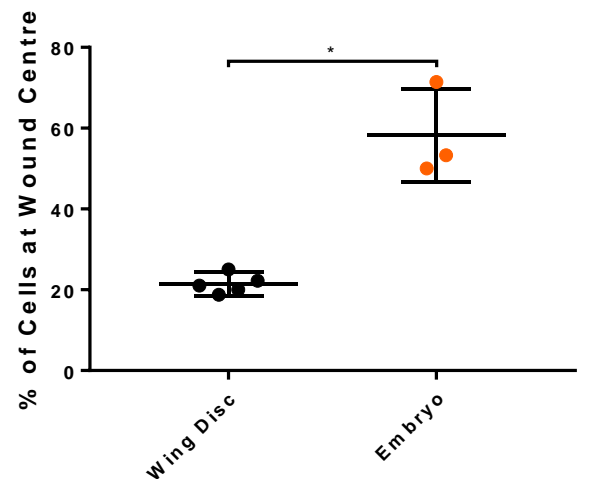


a
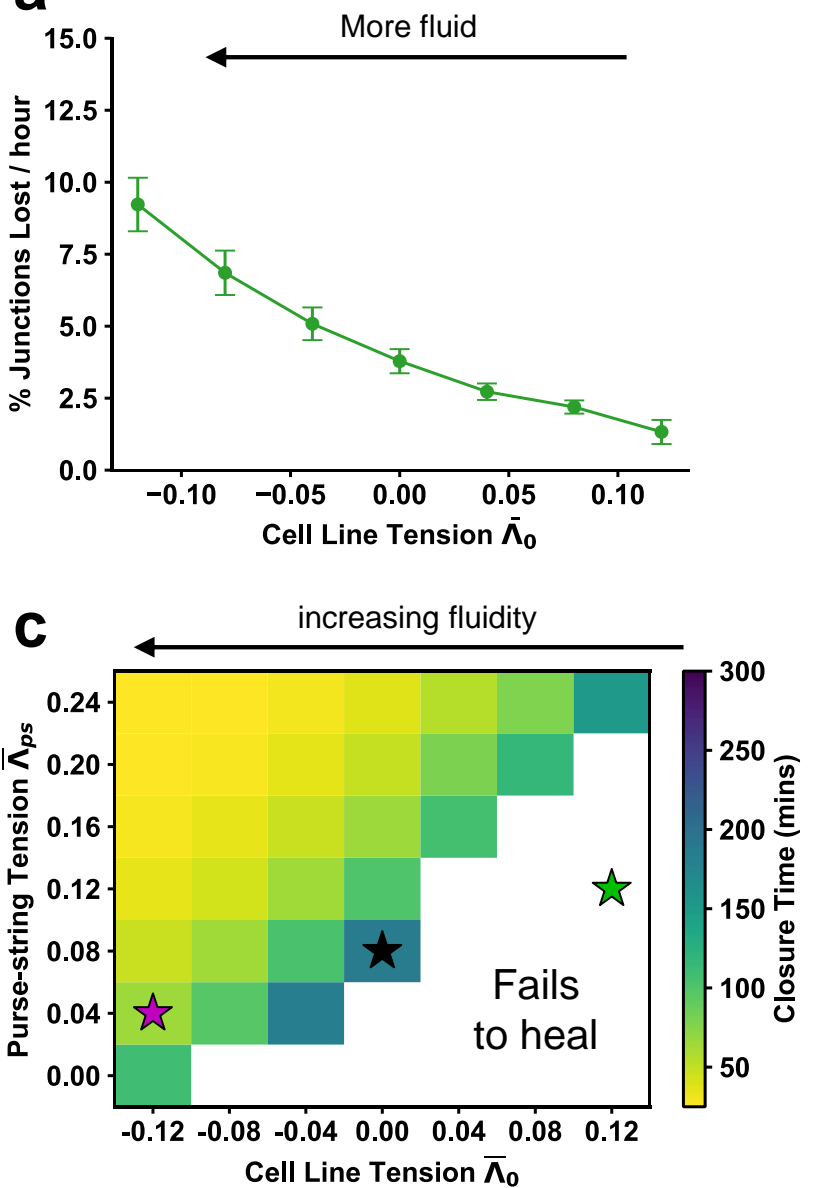

e

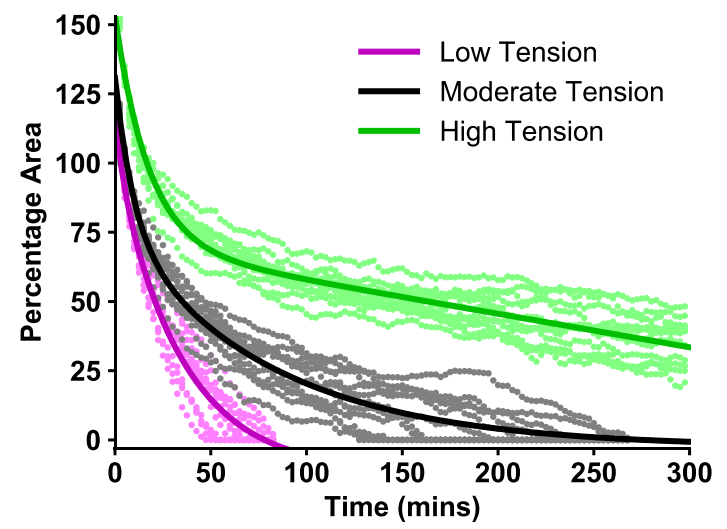

b
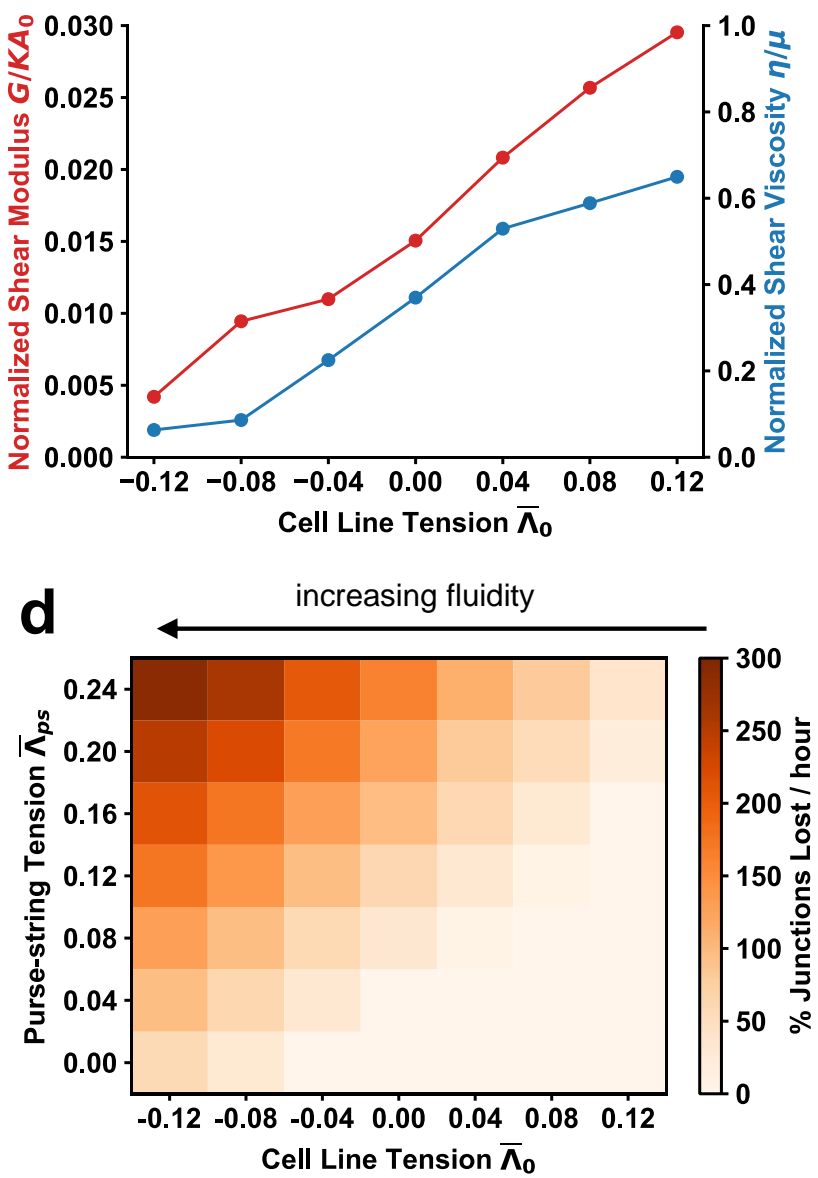

f

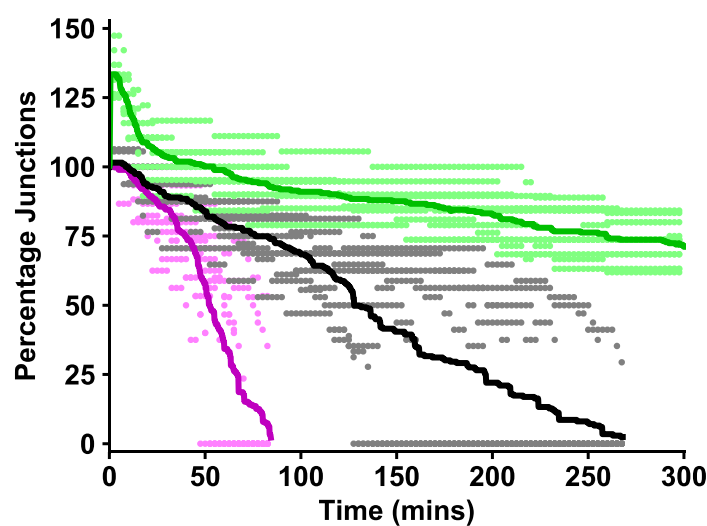


a

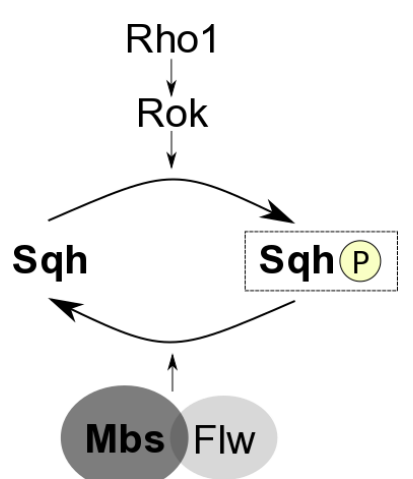

Myosin Phosphatase

\section{C $r n>M b s$ RNAi}
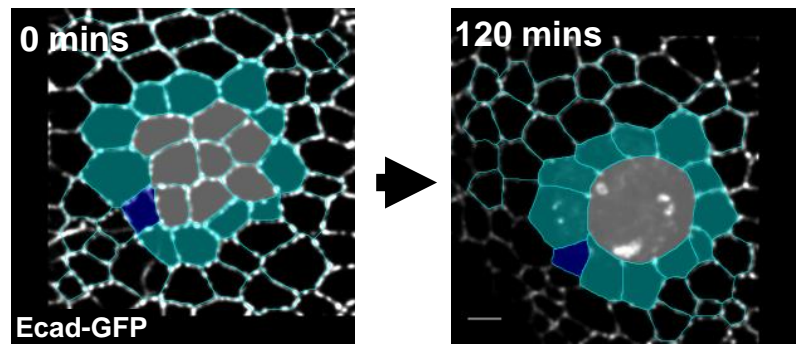

e

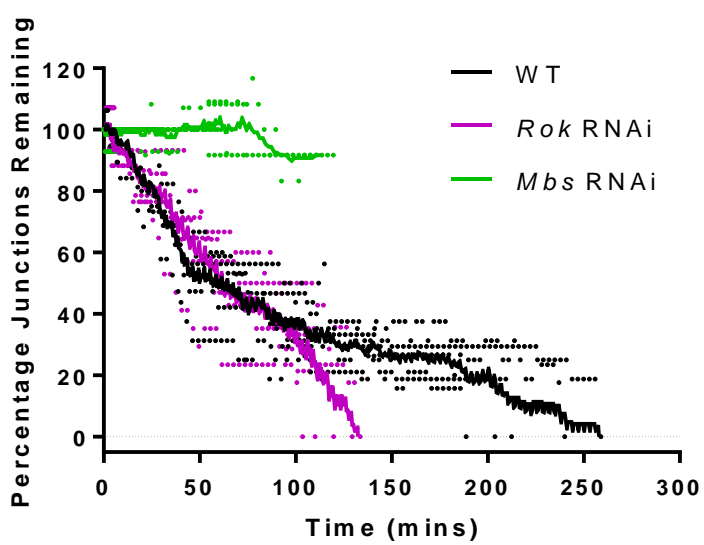

g

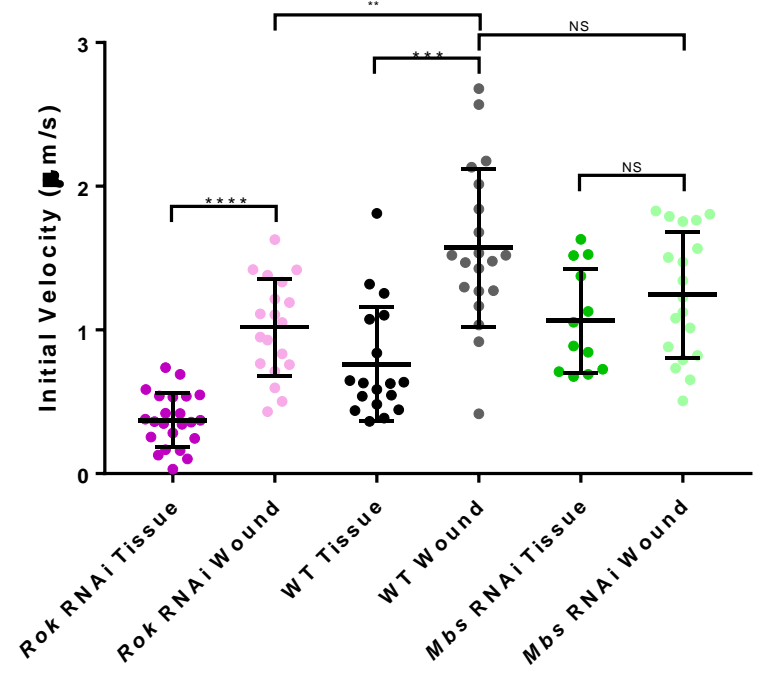

b

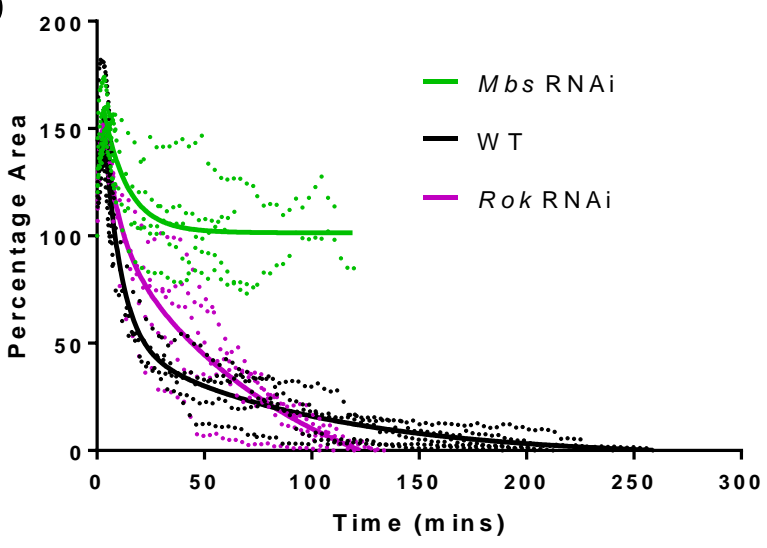

d

$r n>$ Rok RNAi
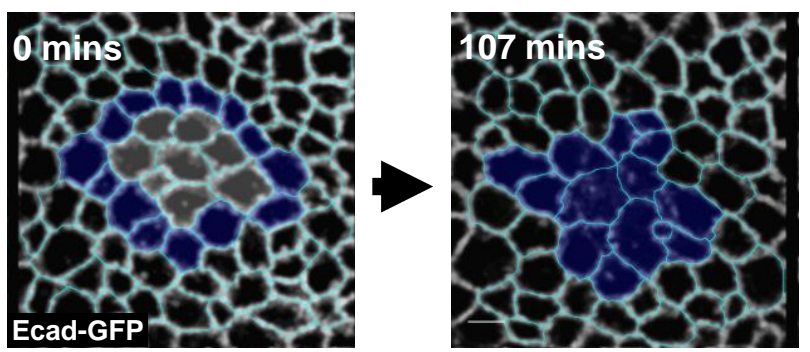

f

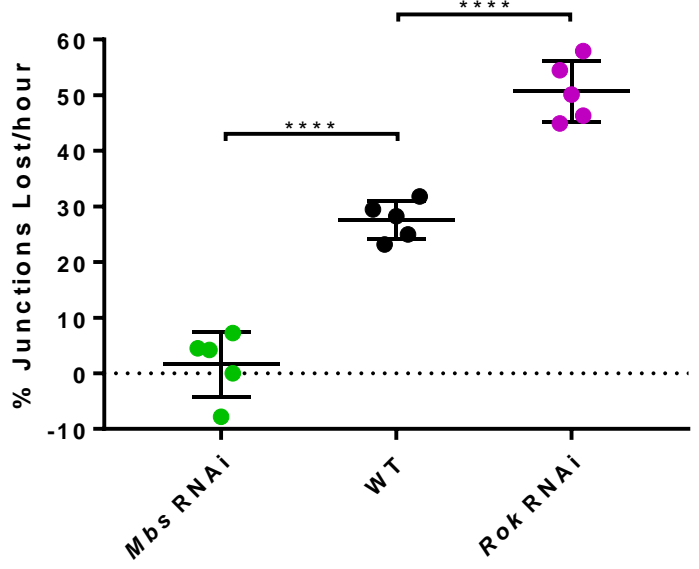

h
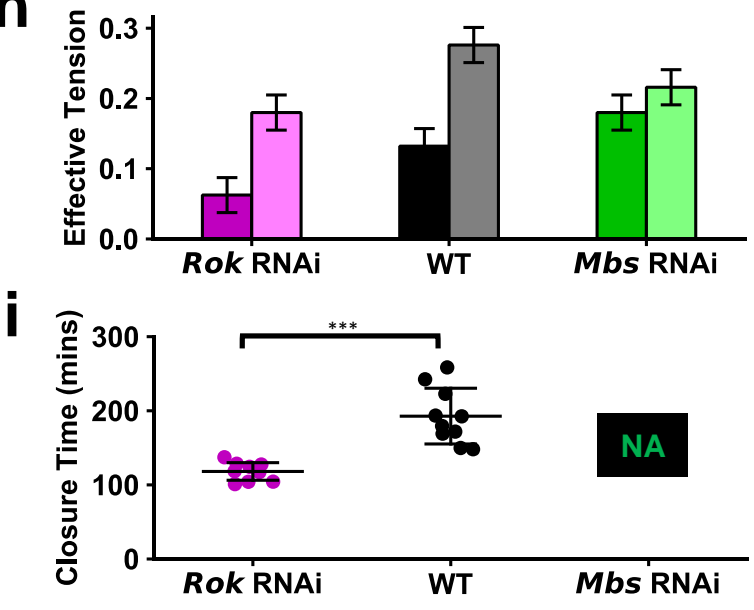
Supplementary Information

Tissue Fluidity Promotes Epithelial Wound Healing

Robert J. Tetley, Michael F. Staddon, Davide Heller, Andreas Hoppe, Shiladitya Banerjee, Yanlan Mao

\section{Contents}

Methods

Page 1-11

Supplementary Figures 1-10

Page 12-23

Supplementary Table 1

Page 23

Supplementary Video Legends 1-7

Page 24-25 


\section{Methods}

\section{Drosophila strains}

Drosophila stocks were raised on conventional cornmeal media at $25^{\circ} \mathrm{C}$. The following alleles and transgenes were used experimentally: shg-GFP (II, referred to as Ecad-GFP), sqh-mCherry ${ }^{2}$ (III), rn-GAL4 (III, MiMIC insertion), UAS-Rok-RNAi (II, VDRC KK Library), UAS-Mbs-RNAi (II, VDRC KK Library), sqh-GFP ${ }^{3}$ (II), shgtdTomato ${ }^{1}$ (II, referred to as Ecad-tdTomato), $s q h^{A X 3}(X)^{4}$, ubi-shg-GFP (II, referred to as ubi-Ecad-GFP) ${ }^{5}$. The experimental genotypes used and their corresponding data are shown in Table 1.

\begin{tabular}{|c|c|}
\hline Genotype & Corresponding Figures and Videos \\
\hline sqhaX3; sqh-GFP, Ecad-tdTomato & Fig. 1a, c, Fig. 3a, Supplementary Video \\
\hline $\begin{array}{l}y w ; \text { Ecad-GFP/+; rn-GAL4/+ (referred to } \\
\text { as WT) }\end{array}$ & $\begin{array}{l}\text { Fig. 1b, e-g, Fig. 3b-g, i, Fig. 5b, e-g, } \\
\text { Supplementary } 4 \text { Fig. } \\
\text { Supplementary Fig. 3a, Supplementary } \\
\text { Fig. 4e, Supplementary Fig. 6a-d, } \\
\text { Supplementary } \quad \text { Figure } \quad 9 b-c \text {, } \\
\text { Supplementary Video 2, Supplementary } \\
\text { Video } 5\end{array}$ \\
\hline Ecad-GFP; sqh-mCherry, pnr-GAL4 & Fig. 1d \\
\hline$y w ;$ Ecad-tdTomato & Fig. 3h-i \\
\hline $\begin{array}{l}y w ; \quad \text { Ecad-GFP/UAS-Mbs-RNAi; } r n- \\
\text { GAL4/+ (referred to as Mbs-RNAi) }\end{array}$ & $\begin{array}{lcr}\text { Fig. 5b-c, e-g, } & \text { Supplementary } & \text { Fig. } 4 \mathrm{e}, \\
\text { Supplementary } & \text { Fig. } & 6 \mathrm{a}-\mathrm{d}, \\
\text { Supplementary } & \text { Fig. } & 7 \mathrm{a}-\mathrm{e}, \\
\text { Supplementary } & \text { Fig. } & 9 \mathrm{~b}-\mathrm{c}, \\
\text { Supplementary Video } 6 & \end{array}$ \\
\hline $\begin{array}{l}\text { yw; } \quad \text { Ecad-GFP/UAS-Rok-RNAi; } \\
\text { GAL4/+ (referred to as Rok-RNAi) }\end{array}$ & $\begin{array}{lcr}\text { Fig. 5b, d-g, Supplementary } & \text { Fig. } 4 \mathrm{e}, \\
\text { Supplementary } & \text { Fig. } & 6 \mathrm{a}-\mathrm{d}, \\
\text { Supplementary } & \text { Fig. } & 8 \mathrm{a}-\mathrm{e}, \\
\text { Supplementary } & \text { Fig. } & 9 \mathrm{~b}-\mathrm{c} \text {, } \\
\text { Supplementary Video } 7 & \end{array}$ \\
\hline w; ubi-Ecad-GFP & $\begin{array}{l}\text { Supplementary } \quad \text { Fig. } \\
\text { Supplementary Fig. 9a-h }\end{array}$ \\
\hline
\end{tabular}

Table 1. Drosophila experimental genotypes and corresponding data

\section{Live imaging of wing imaginal discs}

Late third instar wing imaginal discs were cultured in Shields and Sang M3 media (Sigma) supplemented with $2 \%$ FBS (Sigma), 1\% pen/strep (Gibco), 3ng/ml ecdysone (Sigma) and $2 \mathrm{ng} / \mathrm{ml}$ insulin (Sigma). Wing discs were cultured in $35 \mathrm{~mm}$ fluorodishes (WPI) under $12 \mathrm{~mm}$ filters (Millicell), as described elsewhere ${ }^{6}$. Wing discs were imaged on a Zeiss LSM 880 microscope with Airyscan at $512 \times 512$ resolution with a $63 x$ objective (NA 1.4 ) at $5 x$ zoom. Laser power of $0.2-0.3 \%$ was used and images were captured with a $0.5 \mu \mathrm{m}$ z-spacing. Time intervals varied depending on the experiment. For single channel wounding experiments, the first 25 images were captured with no time interval (to visualise the fast, early dynamics of 
wound closure, using a sufficient z-stack depth to include all cells in the field of view). For two channel wounding experiments, the first 12 images were captured with no time interval. Subsequent imaging was then performed with a time interval of 3 minutes for both single and two channel experiments, with a total z-stack depth of approximately $40 \mu \mathrm{m}$, until wounds closed. For experiments measuring unwounded intercalation rates, a time interval of 3 minutes was used for a total imaging time of 2 hours.

\section{Wounding of tissues}

Wing discs were wounded using a pulsed Chameleon Vision II TiSa laser (Coherent), tuned to $760 \mathrm{~nm}$. Ablation was performed on small, manually defined circular regions of interest (ROIs) that coincided with the tricellular junctions shared between all cells to be ablated. This was necessary, as larger ROls produced significant autofluorescent scarring that made subsequent image analysis impossible. Furthermore, ablation of larger regions led to cavitation, which masked the initial recoil dynamics of the wound. Ablation was performed in a single z-plane, at the level of the adherens junctions.

\section{Segmentation and tracking of wound time-lapse images}

Ecad time-lapse images were first deconvolved using Huygens (Scientific Volume Imaging). Deconvolved images were segmented, tracked and analysed using EpiTools ${ }^{7}$. The following MATLAB-based analysis modules were implemented in the following order: Adaptive projection, Contrast Enhancement (CLAHE), Cell segmentation, Automatic seed tracking, Re-segmentation, Generate skeletons. The adaptive projection method originally developed for EpiTools ${ }^{7}$ was extended through an additional plugin that allowed the user to interactively control and guide the automated creation of the projection surface. This was necessary in regions with strong peripodial membrane signal just above the main layer of cells or in regions with strong surface gradients which could lead to a misaligned surface. The interactive tool suggested surface markers at locations with sufficient cell signal intensity in the 3D image volume. Additional markers could be added or removed by the user. The markers were used to guide the surface fitting procedure in EpiTools ${ }^{7}$ by providing a first estimate of the projection surface location which would be refined in a second iteration of the surface fitting using the cell signal intensities in its local vicinity. Cell signal intensities along the fitted surface within the 3D image volume formed the final projection image. The settings used for "Adapative projection" and "Cell segmentation" were selected on a case-by-case basis, to give the best result for each time-lapse image. Default settings were used for "Contrast Enhancement (CLAHE). "Automatic seed tracking" was implemented so that each cell had a single seed, where possible. Corrected seeds were used for "Re-segmentation" and the resulting corrected segmentation was used to "Generate skeletons". The remaining segmentation and tracking was performed using EpiTools' Icy Plugins. Skeletons were manually corrected using "CellEditor". The corrected skeletons were used to implement "CellGraph", which was run using the "STABLE_MARRIAGE" algorithm for tracking with a Propagation Limit of 5 frames without cutting any border lines. To allow manual tracking correction, an additional helper tool was developed as part of the EpiTools ${ }^{7}$ plugins for icy (code and executable of this customized version of the plugins for Icy are publicly available at: github.com/epitools/manual_tracking). The correction functionality is accessible through the TEST tool in the $\bar{C}$ ellOverlay icy plugin. The new user interface guides the user in the correction process by 
visualizing a superimposition of two consecutive time points (frames). This feature is implemented by displaying the segmentation skeleton of the previous frame $(\mathrm{t}-1)$ on top of the current frame $(\mathrm{t})$ and highlighting the cell to be linked in the skeleton. The user can link the highlighted cell by clicking on any cell in the current frame $(\mathrm{t})$. Optionally the user can also choose to connect the current cell track to another existing track (i.e. merge the two tracks into one) or register a cell division or cell elimination event. The underlying procedure saves any tracking correction to an external file as backup and merges modifications with existing tracking information, from EpiTools itself or other sources.

\section{Quantitative analysis of wound time-lapse images}

The following data was quantified and exported using the "CellOverlay" Icy plugin in EpiTools ${ }^{7}$. Statistical analysis and curve fitting was performed in Prism (GraphPad):

Wound area - the killed cells and resulting wound were manually selected using the "CELL_COLOR_TAG" tool and data exported. Wound area was then expressed as a percentage of the total area of all the killed cells.

Wound edge junctions remaining - the initial number of wound edge cells was counted manually prior to wounding. The polygon number of the wound was quantified using the "CELL_COLOR_TAG" tool. The wound polygon number was expressed as a percentage of the initial number of wound edge cells. When percentage of junctions remaining data was pooled from multiple wing discs a moving average curve ( \pm 4 time points) was fitted to the data.

Distinction of fast and slow closure phases - a two-phase exponential decay curve was fitted to the cell area data pooled from multiple wing discs. The fast and slow phases of closure are represented by the ranges of the fast and slow exponential decays respectively.

Intercalation rates - an intercalation was defined as the loss of a cell-cell junction that was subsequently replaced by a new junction in the orthogonal direction. When quantifying such events, two rules were introduced: 1) to buffer against segmentation errors that lead to the false identification of an intercalation, only intercalations in which the new junction persisted for at least 6 minutes were scored, 2) as junctions were roughly 12 pixels in width, the segmentation software was unable to accurately recognise very short junctions or transient 4-way vertices. Therefore, any junction under 6 pixels in length was assigned a length of 0 , meaning that cells were considered to be in a 4-way vertex configuration. To extract intercalation data, the "EDGE_T1_TRANSITIONS" tool was run and data exported. To enable the tracking of T1 transitions at a better granularity, the edge tracking framework in EpiTools ${ }^{7}$ was rewritten to make it possible to track edges from a frame of choice. Several output statistics were added to the data export, including a summary report for the number of edges and $\mathrm{t} 1$ transitions in each frame. The visualization of the "EDGE_T1_TRANSITIONS" tool was also improved by labelling individual edges with respect to their tracking status. The code and executable for all the discussed modifications is included in the customized version linked above. To quantify intercalations in the tissue (rather than at the wound edge), "EDGE_T1_TRANSITIONS" was initialised in the first time point. To quantify intercalations at the wound edge, "EDGE_T1_TRANSITIONS" was initialised in the 
second time point, after the wound had been generated. The wound itself was treated as a cell, meaning an intercalating tetrad was formed of three wound edge cells and the wound. Intercalation analysis was performed only on junctions that were present in the time point at which "EDGE_T1_TRANSITIONS" was initialised. Junction loss events were scored in the time point immediately prior to the formation of a stable new junction. At each time point, the number of junctions lost was expressed as a percentage of the total number of junctions. These values were then summed and divided by the total time (in hours) to obtain an intercalation rate expressed as Percentage of Junctions Lost/hour.

Cell Division Rates - the number of cells dividing was exported from the "DIVISIONS_AND_ELIMINATIONS" tool and expressed as a percentage of the total number of cells obtained from the "TRACKING_STABLE_ONLY" tool. This was then divided by the total time of each movie to give Percentage of Cells Dividing/hour.

Polygon distributions of wound edge cells - initial wound edge cells were selected manually using the "CELL_COLOR_TAG" tool, prior to wounding. These identities were propagated through all time points. Data was exported and the polygon distributions quantified by pooling all data from all wound edge cells in all wing discs. Polygon distributions were quantified at time points prior to and immediately after wounding and immediately after closure.

Cell elongation - cell elongation was calculated by dividing the major and minor axis length of best fit ellipses (exported from the "CELL_COLOR_TAG" tool). The mean elongation of pooled cells was calculated prior to wounding. Changes in mean cell elongation were expressed as a percentage of the initial mean elongation.

Overlay images were generated by selecting the relevant "CellOverlay" tool layer.

\section{Myosin II quantification}

Myosin II was quantified during the first hour post wounding of $s q h^{A \times 3}$; sqh-GFP, Ecad-tdTomato wing discs, using Sqh-GFP as a reporter. Because these experiments were performed in a sqh ${ }^{A X 3}$ null background, all molecules of Myosin II were tagged with GFP. First, raw (not deconvolved) Sqh-GFP images were background subtracted using the Rolling Ball tool in FIJI with a radius of 12 pixels. Maximum intensity projections (MIPs) of Sqh-GFP images were then generated, which excluded any signal from the overlying peripodial membrane cells. The CellGraph function of EpiTools was run on the Sqh-GFP MIPs using the segmented skeleton images from the corresponding deconvolved Ecad-tdTomato channel (segmentation performed as above).

Junctions were selected manually using the "EDGE_COLOR_TAG" CellOverlay tool in EpiTools ${ }^{7}$. Edge Intensity Buffer and Vertex Intensity Buffer were set to 3 and Selection Mode 1 was used to exclude vertices from the quantification. Two groups of junctions were selected in each movie; 1) junctions in contact with the wound edge in each frame and 2) 10 junctions in the surrounding tissue that persisted for the entire time window of quantification, were more than one cell diameter away from the wound and were not associated with a cell division event. Mean Sqh-GFP intensities were exported for all tagged junctions, with the mean calculated from the top $90 \%$ of pixel intensities for each junction (to exclude any dark pixels that were outside of the cell's cortex). Wound edge junction intensities were 
normalised to the mean intensity of the 10 junctions in the surrounding tissue for each time point. The relative normalised intensity of wound edge junctions was then calculated by dividing by the mean normalised intensity of wound edge junctions in the time point prior to wounding (t0).

\section{Assigning cell row fates (Fig. 3c-e)}

Cells were assigned row identities using the "CELL_COLOR_TAG" CellOverlay tool in EpiTools". This was done prior to wounding and these identities were propagated in time through the entire movie. Cells could therefore change rows over time (by intercalation), but still retained their initially assigned identities (Supplementary Video $5)$.

\section{Intercalation row analysis (Fig. $\mathbf{3 g}$ )}

An intercalation co-ordinate was calculated for every intercalation event (tissue and wound edge) as the intersect between the centroids of the four intercalating cells or three intercalating cells and the wound, at the time point immediately prior to the formation of a new stable junction. To simplify analysis, the wound was considered as a circle of the same area quantified for the wound at a given time point, giving a standardised wound radius (woundradius). Rows of cells were considered as concentric rings around the central wound, the diameter of each row (rowdiameter) being the mean diameter of cells for a particular movie. Row identities were assigned at each time point, rather than being initialised in the first time point, meaning that cells could change row identities over time (for instance, a row 1 cell could become a row 2 cell after intercalating away from the wound edge). The distance between the wound centroid and intercalation co-ordinate (Icaldistance) was calculated for every intercalation event. To determine which row away from the wound the intercalation fell into, the following calculation was used:

(Ical $^{\text {distance }}$ - wound $\left.{ }^{\text {radius }}\right) /$ row $^{\text {diameter }}$

The value obtained was then rounded up to the nearest integer, which referred to the row number. The number of intercalations in a row, at a particular time point, was scored and divided by the area of the row (which changed over time as the area of the wound reduced). These values were summed and divided by the total time of the movie (in hours) to give an intercalation rate in Intercalations $/ \mathrm{\mu m}^{2} / \mathrm{hour}$.

\section{Vertex model}

We model the apical surface of the tissue as a $2 \mathrm{D}$ network of polygonal cells, with cell-cell interfaces represented by straight edges, and three-way junctions by vertices. The total mechanical energy of the tissue is given by:

$$
E=\sum_{\alpha} \frac{1}{2} K\left(A_{\alpha}-A_{\alpha}^{0}\right)^{2}+\sum_{\alpha} \frac{1}{2} \Gamma P_{\alpha}^{2}+\sum_{\langle i, j\rangle} \Lambda_{i j} l_{i j}
$$

where the individual cells are labelled by $\alpha$, and the edges connecting vertices $i$ and $j$ by $\langle i, j\rangle$ (Supplementary Fig. 2). The first term represents the area elasticity of the cells, with elastic modulus $K, A_{\alpha}$ is the area of cell $\alpha$ and $A_{\alpha}^{0}$ is the preferred area. The second term represents contractile energy of the actomyosin cortex for a cell with perimeter $P_{\alpha}$, and contractile tension, $\Gamma$. The final term represents the energy cost due to line tensions $\Lambda_{i j}$ acting on edges of length $l_{i j}$ which is a combination of 
cell-cell adhesion and cortical tension. Negative line tension implies cell-cell adhesion dominates over cortical tension, such that cells tend to maximize the length of junctions between their neighbors. The net mechanical force acting on the vertex $i$ is given by $\boldsymbol{F}_{i}=-\partial E / \partial \boldsymbol{x}_{i}$. Assuming over-damped dynamics, the equation of motion for vertex $i$ is:

where $\mu$ is the coefficient of friction.

$$
\mu \frac{d \boldsymbol{x}_{i}}{d t}=\boldsymbol{F}_{i}
$$

In this study, we non-dimensionalize energy by $K / A_{0}^{2}$ and length by $A_{0}^{1 / 2}$, resulting in normalized contractility $\bar{\Gamma}=\Gamma / K A_{0}$ and normalized line tension $\bar{\Lambda}=\Lambda / K A_{0}^{3 / 2}$. Time is non-dimensionalized by the $T^{*}=\mu / K A_{0}$. The equation of motion is discretized as:

$\overline{\boldsymbol{x}}_{i}(t+\Delta t)=\overline{\boldsymbol{x}}_{i}(t)+\frac{\Delta t}{T^{*}} \overline{\boldsymbol{F}}_{i}$,

where $\Delta t$ is the timestep. See Table 2 for a complete list of default parameter values. As the tissue relaxes by minimizing mechanical energy, cell edges may shrink due to contractile forces. If an edge length goes below a small threshold length, $L_{T 1}$, an intercalation, or T1 transition, occurs, in which a new edge is formed perpendicular to the original junction, if it results in a lower energy. To keep the system out of equilibrium, we introduce two sources of activity: cell division, and line tension fluctuations, as described below.

Cell division - we implemented a simplified model of the cell cycle, with cells in one of three phases; resting, growing or dividing. Cells start in the resting phase, with the default preferred area $A_{0}$. Once they have reached a threshold age, they transition to mitosis at a fixed rate. In mitosis, the preferred area of the cell doubles over a period of 30 minutes, resulting in growth of the cell. After 30 minutes, the cell is divided into two new cells by creating a new edge between two of the cell edges which is chosen to minimize the system energy. This results in division of elongated cells along their short axis.

Line tension fluctuations - to model myosin II fluctuations, we allow the line tensions to fluctuate over time, adapting the model introduced in Curran et al. ${ }^{9}$.The line tension $\Lambda_{i j}$ on the edge joining vertices $i$ and $j$ evolves over time as:

$$
\frac{d \bar{\Lambda}_{i j}}{d t}(t)=-\frac{1}{\tau_{m}}\left(\bar{\Lambda}_{i j}(t)-\bar{\Lambda}_{0}\right)+\xi_{i j}(t)
$$

where $\tau_{m}$ is a persistence time of myosin, $\bar{\Lambda}_{0}$ is the mean line tension, and $\xi_{i j}$ is an uncorrelated white noise obeying:

$\left\langle\xi_{i j}(t) \xi_{k l}\left(t^{\prime}\right)\right\rangle=\frac{2 \sigma_{m}^{2}}{\tau_{m}} \delta\left(t-t^{\prime}\right) \delta_{i k} \delta_{j l}$.

The line tension deviation, $\sigma_{m}$, controls the amount of fluctuation around the mean, and is equal to the standard deviation of the line tension over time.

\section{Modelling laser ablation and wound healing}

Wounds in the epithelium are created by removing any cell that lies partially or fully within a circle of radius $R_{w}$. As material remains within the wound, but the actomyosin cortices are disrupted, we remove all contractility within the wound. The polygons constituting the wound have an area elasticity term with elastic modulus $K_{w}$ and zero contractility. As tissue material leaves the gap during wound closure, the elastic modulus decreases to zero over 10 minutes. At the same time, tension in the 
purse-string surrounding the wound increases from the mean line tension, $\bar{\Lambda}_{0}$, to $\bar{\Lambda}_{p s}$, the purse-string tension. The resulting effect is a rapid expansion of wound area after ablation, followed by a contraction back to the original size over 10 minutes. We simulate the tissue dynamics for 300 minutes, or until the wound closes.

\section{Model implementation}

The model is implemented using Surface Evolver ${ }^{10} .200$ cells are generated using a Voronoi tessellation and relaxed, without fluctuations and divisions, to a steady state. The simulation is then run, with divisions and fluctuating line tensions, until the tissue has grown to 250 cells. Next, the wound is ablated, and the simulation is run until wound closure or for a maximum of 300 minutes.

\section{Model parameters}

We use the same normalized contractility $\bar{\Gamma}$ as in Farhadifar et al. ${ }^{8}$. Due to differences between our models, such as division rules, the same set of parameters do not apply. The mean division rate is taken from experimental measurements. The wound radius is chosen to ablate the same number of cells as in experiments, and the mean cell area is approximately the cell area measured in experiments. We use the same tension recovery time for line tension $\tau_{m}$ as in Curran et al. ${ }^{9}$. The line tension deviation $\sigma_{m}$, normalized cell tension $\bar{\Lambda}_{0}$, and normalized purse-string tension $\bar{\Lambda}_{p s}$, and friction coefficient $\mu$ are chosen to approximate the WT wounded and unwounded intercalation rates, and wound closure time in experiments, and to capture the slow and fast phases of closure.

\begin{tabular}{|l|c|l|}
\hline Parameter & Symbol & Value \\
\hline Normalized contractility & $\bar{\Gamma}$ & 0.04 \\
\hline Normalized cell line tension & $\bar{\Lambda}_{0}$ & 0.00 \\
\hline Normalized purse string tension & $\bar{\Lambda}_{p s}$ & 0.08 \\
\hline Preferred cell area & $A^{0}$ & $16 \mu \mathrm{m}^{2}$ \\
\hline Friction coefficient & $\mu$ & $30 \mathrm{~s}$ \\
\hline Cell line tension deviation & $\sigma_{m}$ & 0.025 \\
\hline Tension recovery time & $\tau_{m}$ & $150 \mathrm{~s}$ \\
\hline Wound radius & $R_{w}$ & $5.33 \mu \mathrm{m}$ \\
\hline Time step & $\Delta t$ & $3 \mathrm{~s}$ \\
\hline Intercalation threshold length & $L_{T 1}$ & $0.2 \mu \mathrm{m}$ \\
\hline Mean \% Cells Dividing / Hour & & 2.25 \\
\hline
\end{tabular}

Table 2. Default parameters used

\section{Computing tissue elastic modulus and viscosity}

We compute tissue shear modulus and viscosity by performing shear rheology simulations on a model tissue comprised of 400 cells. The tissue is sheared in one time step by a strain $\epsilon$, moving each interior vertex position as $x \rightarrow x+\epsilon y$. With the boundary vertices held fixed, the sheared tissue is then relaxed for a period of 300 minutes (Supplementary Fig. 5a). During shearing, the strain energy density of the tissue, $E / A$, increases, and then relaxes to a non-zero finite value at long times 
(Supplementary Fig. 5b). We obtain the viscoelastic relaxation timescale, $\tau$, and the final strain energy density, $w_{\infty}$, by fitting an exponential function to the computed strain energy density (Supplementary Fig. 5c).

We run shear rheology simulations for $\epsilon$ in the range $0-0.5$ (in steps of 0.025). We compute the shear modulus, $G$, by fitting the equation $w_{\infty}=w_{0}+\frac{1}{2} G \epsilon^{2}$ (Supplementary Fig. 5d). Due to the fluctuations in energy density from tension fluctuations, the estimate for $\tau$ can be noisy. We obtain an estimate for the average viscoelastic timescale by taking the inverse-variance weighted average of $\tau$ over all values of shear strain: $\bar{\tau}=\frac{\sum_{i} \tau_{i} / \sigma_{i}^{2}}{\sum_{i} 1 / \sigma_{i}^{2}}$. Finally, we obtain the tissue viscosity using the relation, $\eta=G \bar{\tau}$.

We verify our calculations by studying the influence of the shape index, $p_{0}=-\frac{\Lambda_{0}}{2 \Gamma}{ }^{11}$, on the continuum materials properties of the tissue. The shape index has been shown to control a fluid to solid jamming transition, with tissues displaying properties of solids when the shape index is below a critical shape index, $p_{0}^{*} \approx 3.81$, and properties of a fluid when above it. We find that as $p_{0}$ increases the tissue fluidizes, with both the shear modulus and the viscosity decreasing to 0 for $p_{0}>3.8$ (Supplementary Fig. 5e-f). Moreover, we observe a bilinear decrease in the elastic modulus and viscosity, with an initial fast decrease for $p_{0}<p_{0}^{*}$, and then a slower decrease for $p_{0}>p_{0}^{*}$, where the shear modulus and viscosity is close to zero.

\section{Comparing model predictions to experimental perturbations}

To compare the model predictions to the WT wing disc, Mbs RNAi, and Rok RNAi mutants, we fit the vertex model parameters using the experimentally measured division rates, and the vertex recoil velocities from laser ablation experiments (Fig. $5 \mathrm{~g}$ ). We assume that the recoil velocity is proportional to the effective junctional tension on each cell.

The effective tension is given by $\bar{\Lambda}^{e f f}=\frac{1}{2} \bar{\Lambda}_{0}+\bar{\Gamma} P^{*}$, and represents the average tension acting on each cell edge at the junction, where $P^{*}$ is the cell perimeter that minimizes the tissue mechanical energy. A factor of half is used as each cell edge contributes to half of the total junction tension. Similarly, the effective purse-string tension is given by $\bar{\Lambda}_{p s}^{e f f}=\bar{\Lambda}_{p s}+\bar{\Gamma} P^{*}$, as only one cell contributes to the line tension term.

For WT, Rok RNAi, and Mbs RNAi tissues, we assume that both the effective tension and the effective purse-string tension are proportional to the initial vertex recoil velocity from laser ablations experiments (Fig. $5 \mathrm{~g}$ ):

$\bar{\Lambda}^{e f f}=\beta v$, and $\bar{\Lambda}_{p s}^{e f f}=\beta v_{p s}$,

where $\beta$ is the scaling factor, $v$ is the mean tissue edge recoil velocity, and $v_{p s}$ is the mean wound edge recoil velocity from experiments. We use the line tension deviation parameter as before, as this reproduces the unwounded intercalation rate measured experimentally.

We treat both the scaling factor, $\beta$, and the friction coefficient, $\mu$, as our fitting parameters, using the same values in all three cases, to approximate the wound healing rates. From the scaling factor, we obtain the cell junction line tension, and 
purse-string tension. The parameters used, that differ from the defaults, are given in Table 3.

\begin{tabular}{|c|l|l|l|}
\hline Parameter & Rok RNAi Value & WT Value & Mbs RNAi Value \\
\hline $\bar{\Lambda}_{0}$ & -0.141 & 0.012 & 0.119 \\
\hline $\bar{\Lambda}_{p s}$ & 0.047 & 0.150 & 0.096 \\
\hline$\mu$ & $80 \mathrm{~s}$ & $80 \mathrm{~s}$ & $80 \mathrm{~s}$ \\
\hline Mean \% Cells Dividing / Hour & 2.240 & 2.274 & 0.8723 \\
\hline
\end{tabular}

Table 3. Model parameters used in Fig. 5h

\section{Live imaging of embryos}

Adult $y w$; Ecad-tdTomato flies were placed in laying cages sealed by agar plates (water 70\% v/v, apple juice 30\% v/v, agar (Sigma) 3\% w/v, methylparaben (Sigma) $0.05 \% \mathrm{w} / \mathrm{v}$ ) coated by a small amount of yeast overnight at $25^{\circ} \mathrm{C}$. Embryos were recovered by rinsing the agar plates with water into a basket. Embryos were dechorionated for $\sim 1$ minute in $12 \%$ sodium hypochlorite solution and rinsed thoroughly with water. Embryos were returned to agar to prevent desiccation and stage $13 / 14$ embryos were selected by eye. Stage 13/14 embryos were affixed to coverslips using heptane glue, with their ventrolateral sides facing the coverslip. Coverslips were then attached to metal slide frames (Leica) using double sided tape and embryos were covered in halocarbon oil 27 (Sigma). Embryos were wounded as above and imaged the same as wing discs, except that 2 minute time intervals were used after the first 25 time points had been acquired. Embryos were allowed to develop to hatching after imaging, to confirm that the imaging process had not been phototoxic. Segmentation of cells was performed as described above.

\section{Comparing intercalation in embryos and wing discs}

As a measure of how much wound edge intercalation had occurred during embryonic and wing disc wound closure, the percentage of cells remaining close to the centre of the wound was quantified. A circle $5 \%$ the area of the original wound was drawn where the centre of the wound was immediately prior to closure. Any cell that intersected this circle immediately after wound closure was scored as having not intercalated away from the wound. The number of cells was then expressed as a percentage of the total number of starting wound edge cells.

\section{Single junction ablations}

Nanoablation of single junctions was performed to provide a measure of junctional tension ${ }^{8}$. Wing discs were mounted as for wounding experiments and imaged using the same microscope. Narrow rectangular ROls were drawn across the centre of single junctions and this region was ablated using the same settings used to wound wing discs (see above). Wing discs were imaged continuously in a single plane using identical settings as described above, except that at least 10x zoom was used. The initial recoil rate of vertices at the ends of ablated junctions was quantified by measuring the change in distance between the vertices and dividing by the initial time step. In unwounded discs, no more than two separate junction ablations were performed in each disc, a large distance apart. Ablations in wound edge junctions 
were performed 15 minutes after wounding, which corresponded to the time at which myosin II intensity reached its peak. A single junction was ablated in each wound edge.

\section{Statistical Information}

The results of all statistical test are thoroughly reported in figure legends. Appropriate statistical tests were chosen based on data distributions. KolmogorovSmirnov and $t$-tests were two-tailed. For biological experiments, replicates represent wing imaginal discs from different animals. Because the segmentation and tracking process is extremely labour intensive, for each genetic condition 5 replicates were used. This was deemed sufficient, as clear statistical differences could be observed between genotypes. 12 replicate vertex model simulations were run for each parameter set.

\section{Data Availability Statement}

The data that support the findings of this study are available from the corresponding author upon reasonable request.

\section{References}

1 Huang, J., Zhou, W. K., Dong, W., Watson, A. M. \& Hong, Y. Directed, efficient, and versatile modifications of the Drosophila genome by genomic engineering. $P$ Natl Acad Sci USA 106, 8284-8289, doi:10.1073/pnas.0900641106 (2009).

2 Martin, A. C., Kaschube, M. \& Wieschaus, E. F. Pulsed contractions of an actin-myosin network drive apical constriction. Nature 457, 495-U411, doi:10.1038/nature07522 (2009).

3 Royou, A., Field, C., Sisson, J. C., Sullivan, W. \& Karess, R. Reassessing the role and dynamics of nonmuscle myosin II during furrow formation in early Drosophila embryos. Mol Biol Cell 15, 838-850, doi:10.1091/mbc.E03-060440 (2004).

4 Jordan, P. \& Karess, R. Myosin light chain-activating phosphorylation sites are required for oogenesis in Drosophila. Journal of Cell Biology 139, 18051819, doi:DOI 10.1083/jcb.139.7.1805 (1997).

5 Oda, H. \& Tsukita, S. Real-time imaging of cell-cell adherens junctions reveals that Drosophila mesoderm invagination begins with two phases of apical constriction of cells. J Cell Sci 114, 493-501 (2001).

6 Zartman, J., Restrepo, S. \& Basler, K. A high-throughput template for optimizing Drosophila organ culture with response-surface methods (vol 140, pg 667, 2013). Development 140, 2848-2848, doi:10.1242/dev.098921 (2013).

7 Heller, D. et al. EpiTools: An Open-Source Image Analysis Toolkit for Quantifying Epithelial Growth Dynamics. Dev Cell 36, 103-116, doi:10.1016/j.devcel.2015.12.012 (2016).

8 Farhadifar, R., Roper, J. C., Algouy, B., Eaton, S. \& Julicher, F. The influence of cell mechanics, cell-cell interactions, and proliferation on epithelial packing. Current Biology 17, 2095-2104, doi:10.1016/j.cub.2007.11.049 (2007).

9 Curran, S. et al. Myosin II Controls Junction Fluctuations to Guide Epithelial Tissue Ordering. Dev Cell 43, 480-492 e486, doi:10.1016/j.devcel.2017.09.018 (2017). 
10 Brakke, K. A. The Surface Evolver. Experimental Mathematics 1, 141-165, doi:10.1080/10586458.1992.10504253 (1992).

11 Bi, D. P., Lopez, J. H., Schwarz, J. M. \& Manning, M. L. A densityindependent rigidity transition in biological tissues. Nat Phys 11, 1074-+, doi:10.1038/Nphys3471 (2015). 

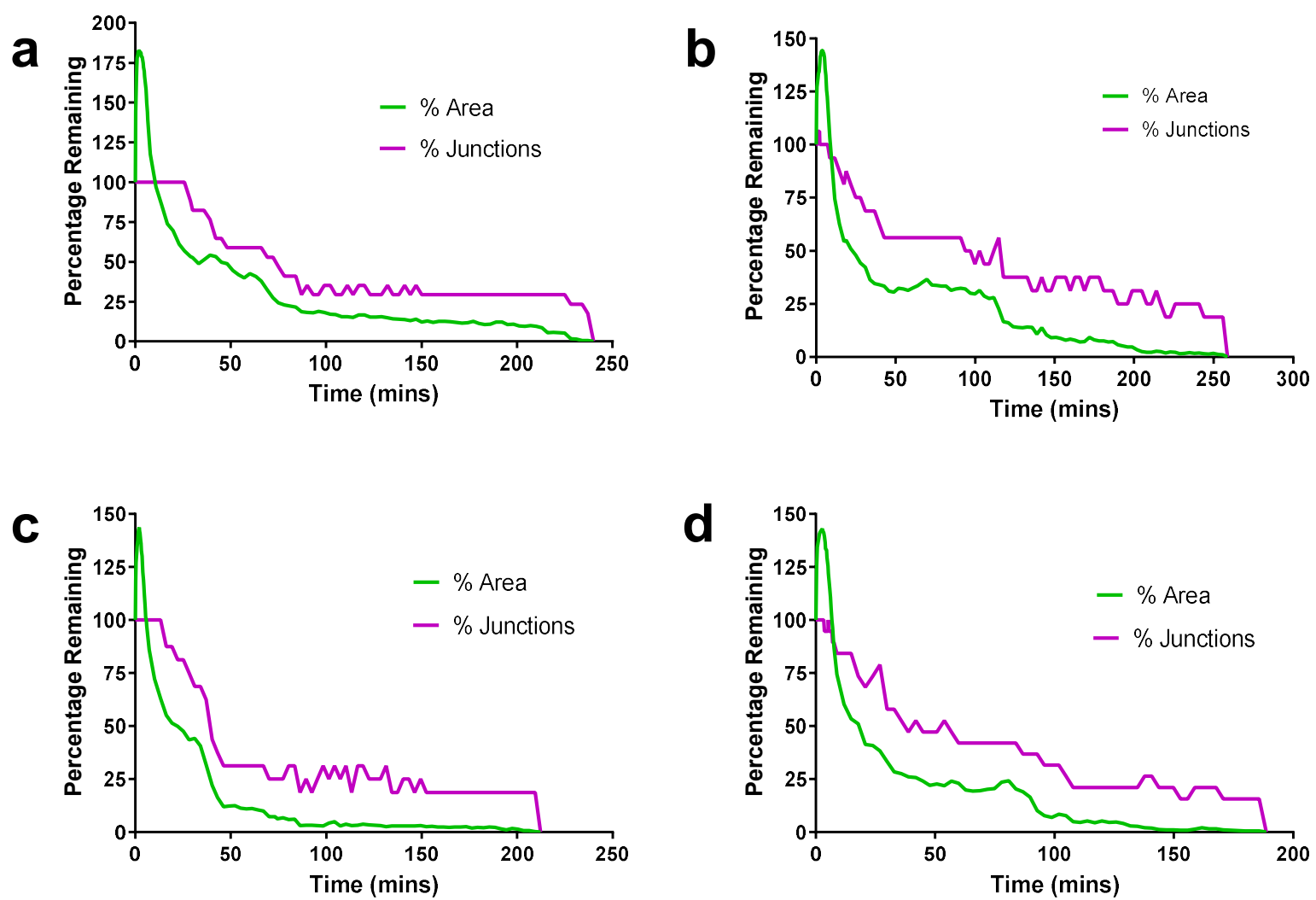

\section{Supplementary Figure 1. Relationship between wound area changes and intercalation events in WT wing discs}

a-d, Quantification of the percentage of starting wound edge junctions (magenta) and wound percentage area (green) for four Ecad-GFP wing disc wounds (in addition to the wing disc in Fig. 1e). 
a $E=\underbrace{\sum_{\alpha} \frac{1}{2} K\left(A_{\alpha}-A_{\alpha}^{0}\right)^{2}}_{\text {Elasticity }}+\underbrace{\sum_{\alpha} \frac{1}{2} \Gamma P_{\alpha}^{2}}_{\text {Contractility }}+\underbrace{\sum_{\langle i, j\rangle} \Lambda_{i j}(t) L_{i j}}_{\text {Line Tension }}$
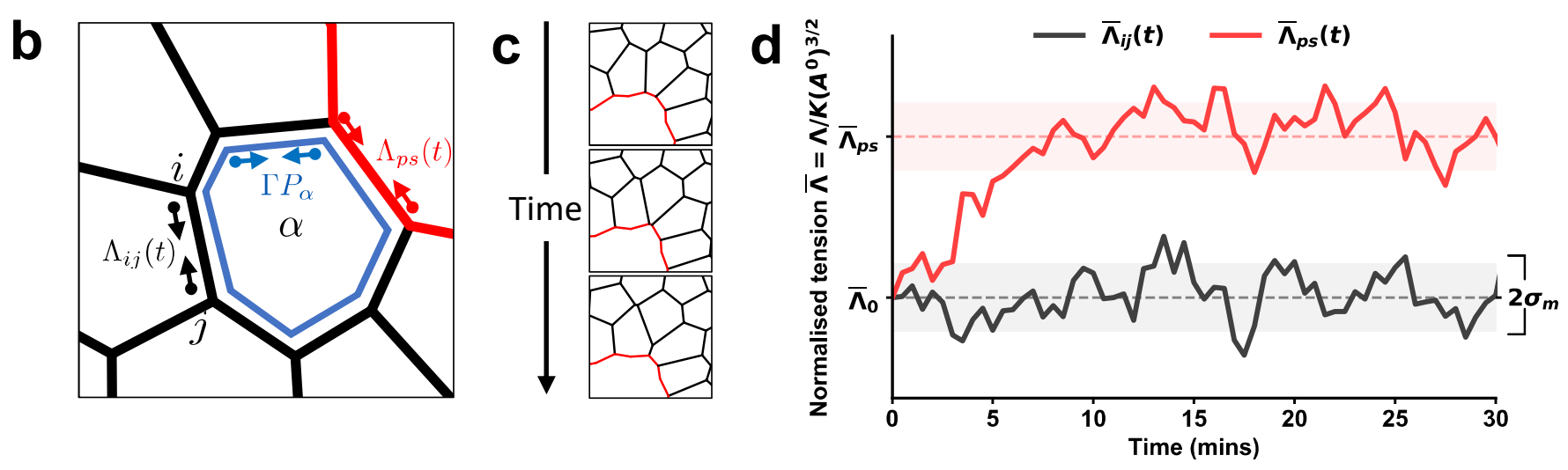

\section{Supplementary Figure 2. Vertex modelling of wound healing}

a, Equation for the total energy of the system. b. Schematic showing the forces due to line tension and contractility acting on a cell in the vertex model. c, Example of a wound edge intercalation. $\mathbf{d}$, Cell edge line tension fluctuation (black) and purse-string tension on the wound (red) over time after ablation ( $T=0$ mins). 

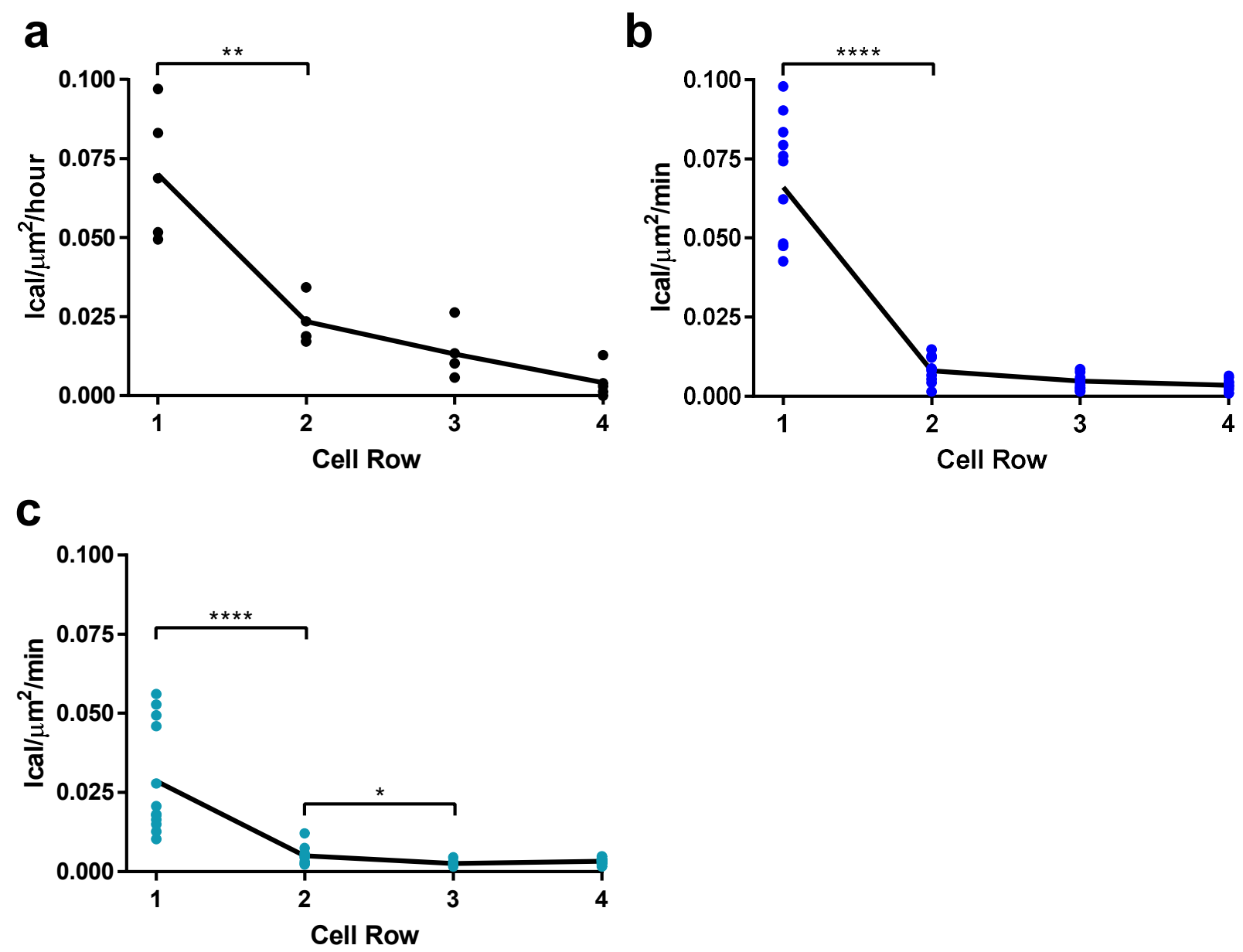

Supplementary Figure 3. Analysis of intercalation rate in rows of cells away from the wound

a-c, Intercalation rates expressed as the number of intercalations per $\mu \mathrm{m}^{2}$ per min for (a) WT wing discs and vertex model simulations with (b) high or (c) low purse string tensions in rows of cells away from the wound edge. Statistically significant differences between neighbouring rows are shown. The intercalation rate was significantly higher in row 1 than row 2 for WT wing discs (Kolomogorov-Smirnov Test, $D=1, p=0.0079$ ), high purse string (Kolomogorov-Smirnov Test, $D=1, p<0.0001$ ) and low purse string (Kolomogorov-Smirnov Test, $\mathrm{D}=0.9167, p<0.0001$ ) simulations. In low purse string simulations, the intercalation rate was also significantly higher in row 2 than row 3 (Kolomogorov-Smirnov Test, $\mathrm{D}=0.5833, p=0.0337$ ). 
a

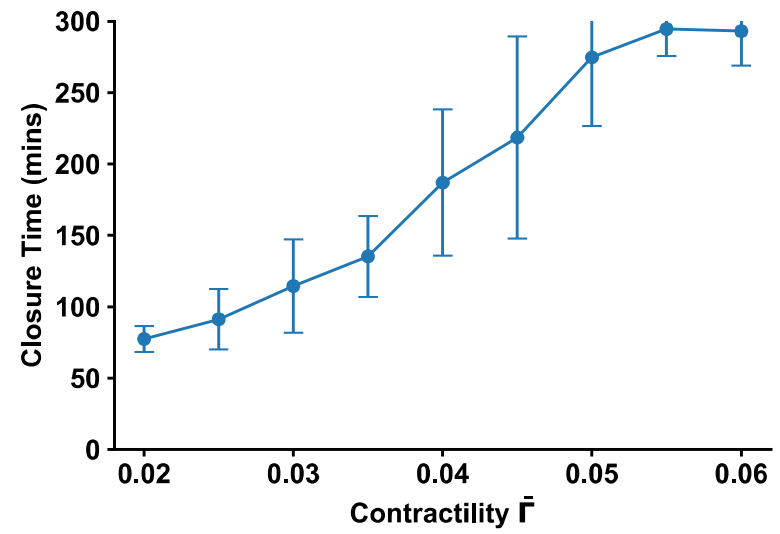

C

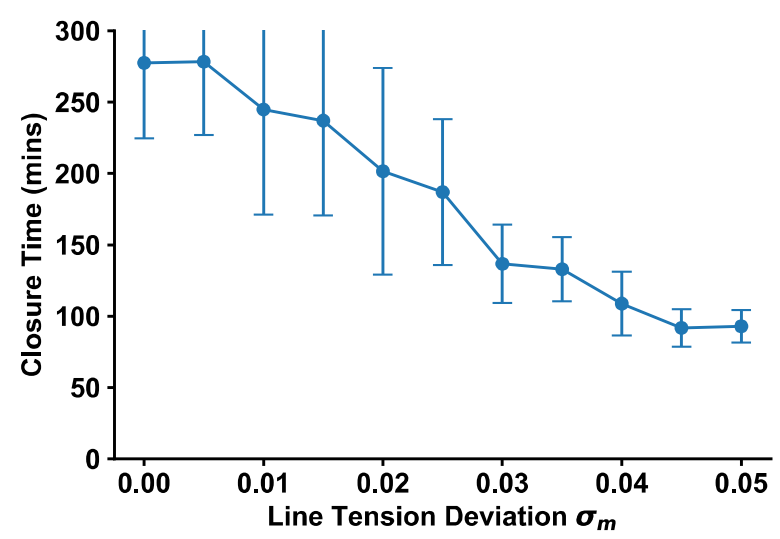

$\mathbf{e}$

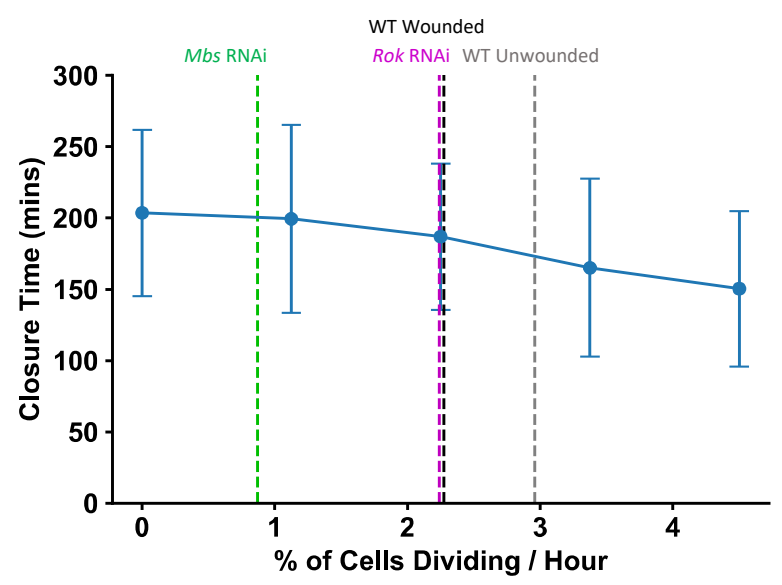

b

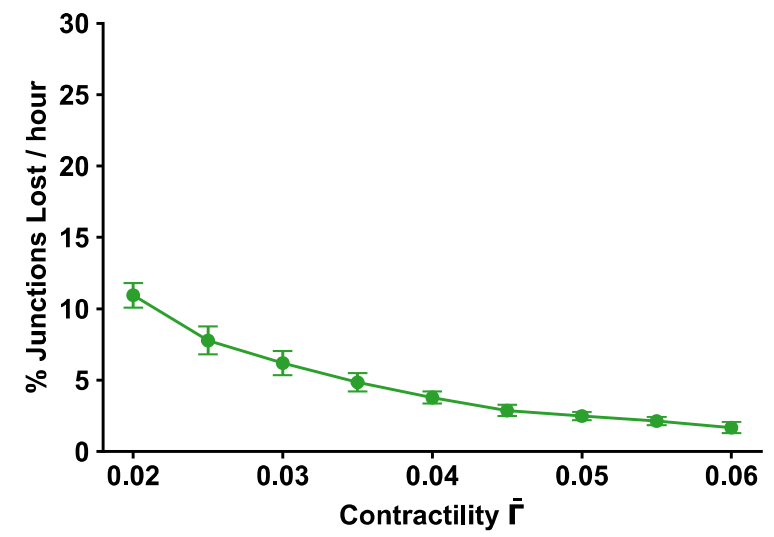

d

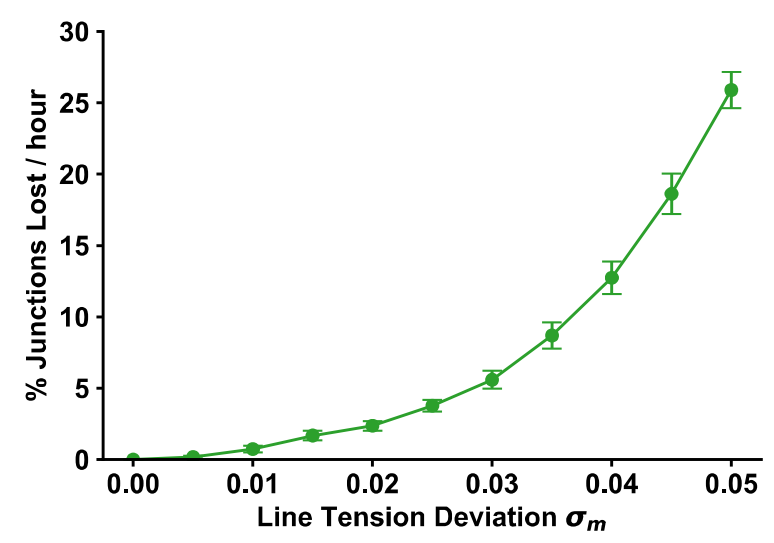

$\mathbf{f}$

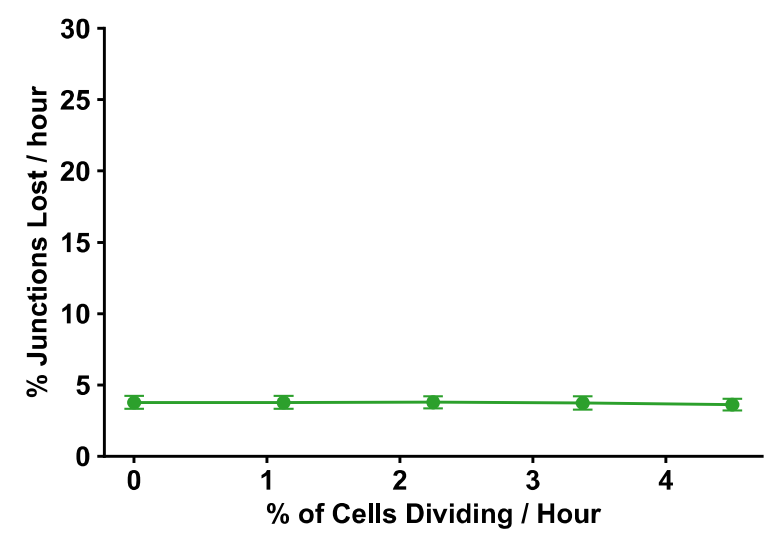

\section{Supplementary Figure 4. Effect of model parameters on fluidity and closure time}

a, Mean intercalation rate in a wounded tissue, and $\mathbf{b}$, mean closure time against normalized contractility. c, Mean intercalation rate in a wounded tissue, and $\mathbf{d}$, mean closure time against cell line tension deviation. e, Mean intercalation rate in a wounded tissue, and $\mathbf{f}$, mean closure time against cell division rate. For each value, $(n=12)$. Dashed lines indicate experimentally measured values of division rates. 
a

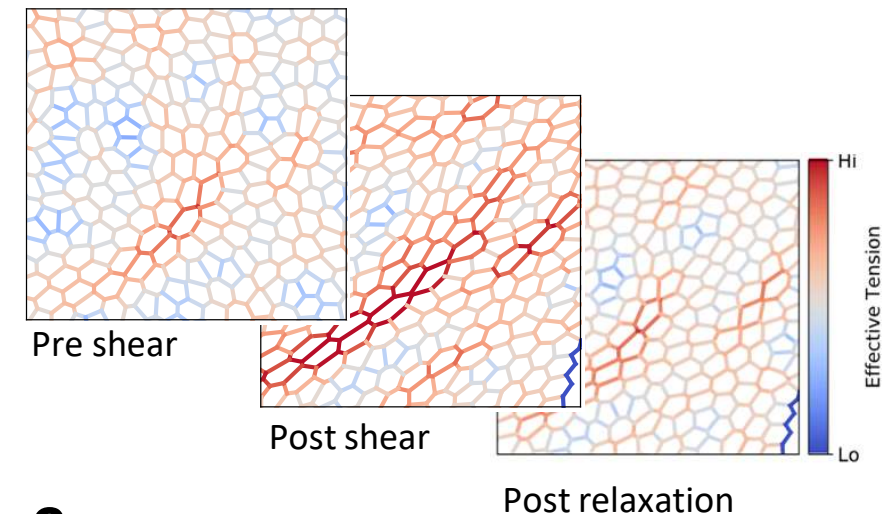

C

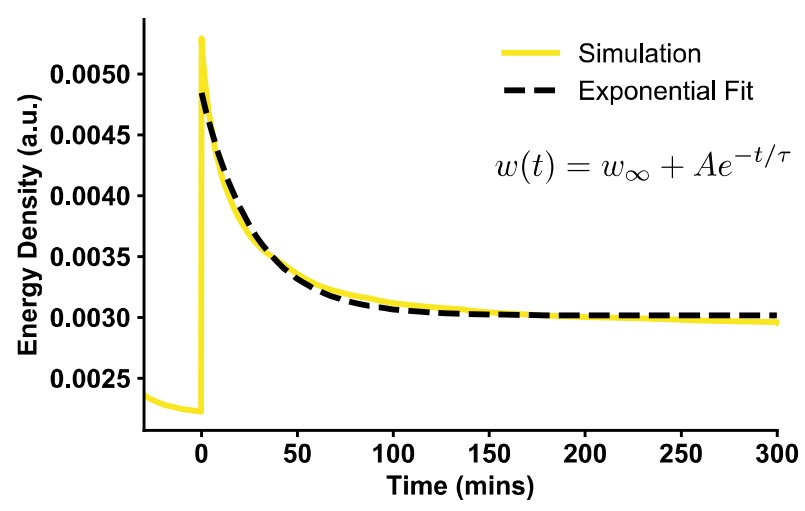

e

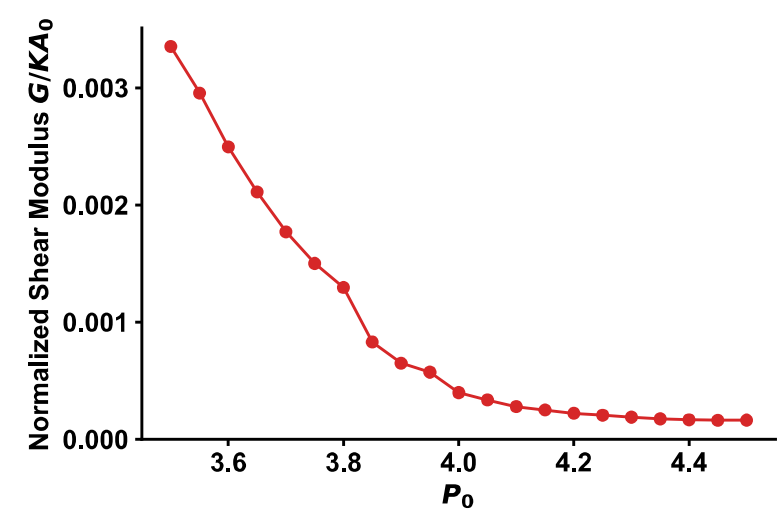

b

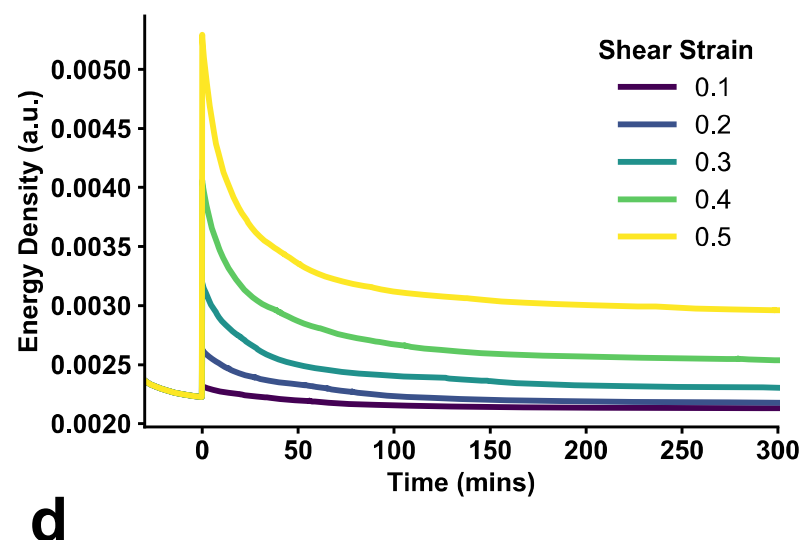

d

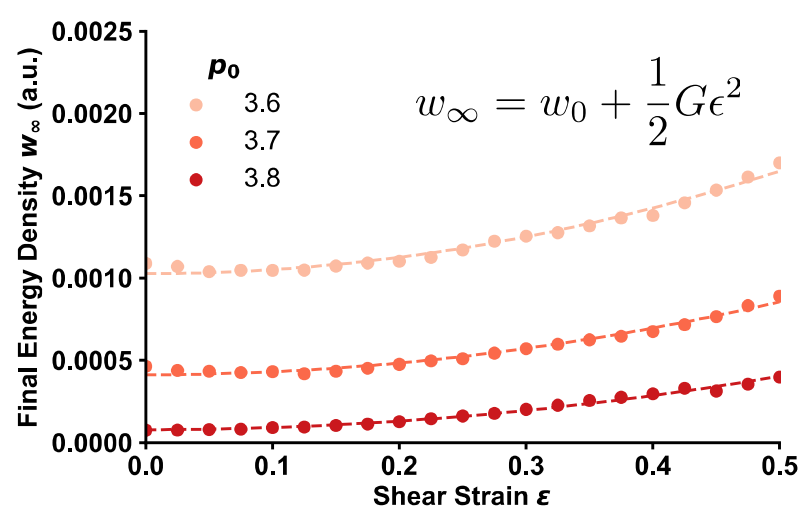

f

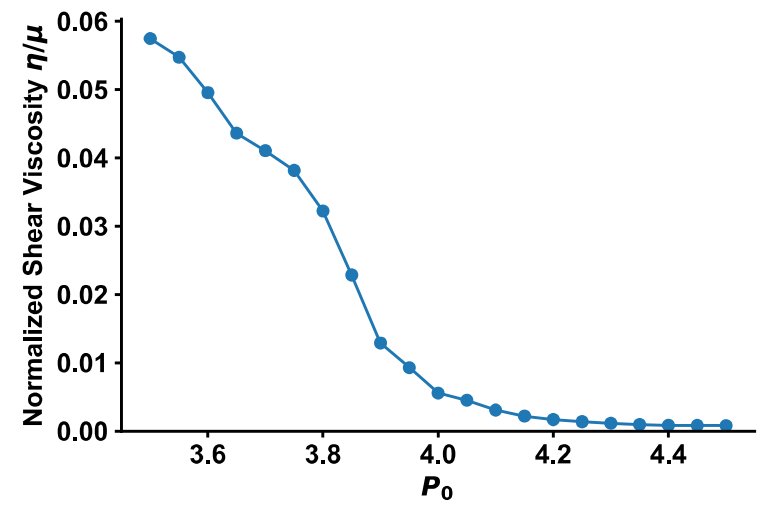




\section{Supplementary Figure 5. Obtaining continuum tissue properties by shearing}

a, Images of a tissue during shearing simulations, before shearing, after shearing, and after relaxation. Edge color indicates the effective tension, the total of the line tension and contractility, on each edge, with blue being low and red high. $\mathbf{b}$, The energy density of the tissue over time, for different amounts of shear strain. c, An exponential curve is fit to the energy density, giving a relaxation time scale, $\tau$, and a final strain energy, $w_{\infty}$. d, Final energy density, $w_{\infty}$, against shear strain, for different values of $p_{0}$. The shear strain is obtained by fitting a quadratic curve. e, The normalized tissue shear modulus against cell shape index $p_{0}$. f, The normalized tissue viscosity against cell shape index $p_{0}$. 
a

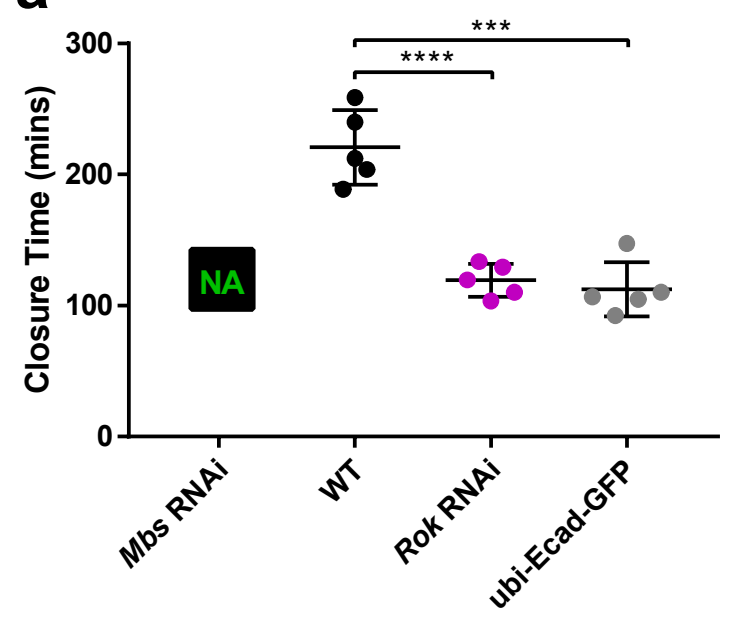

C

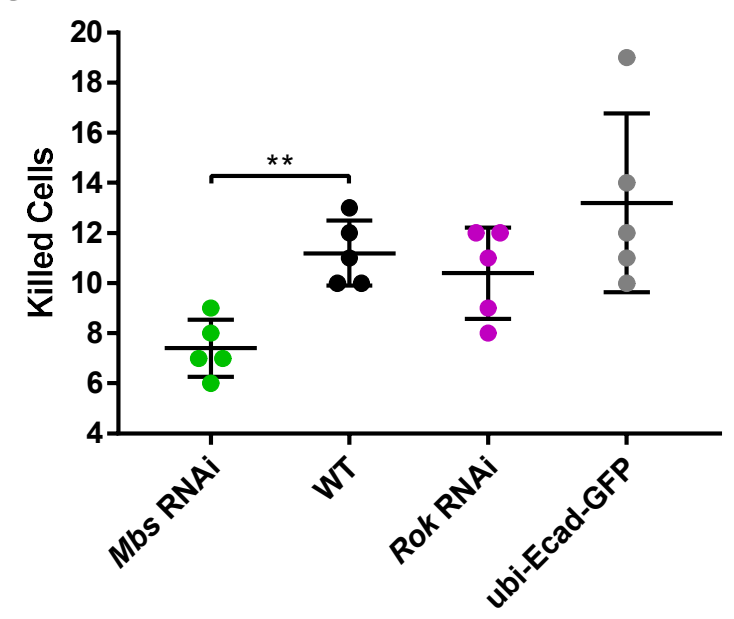

b

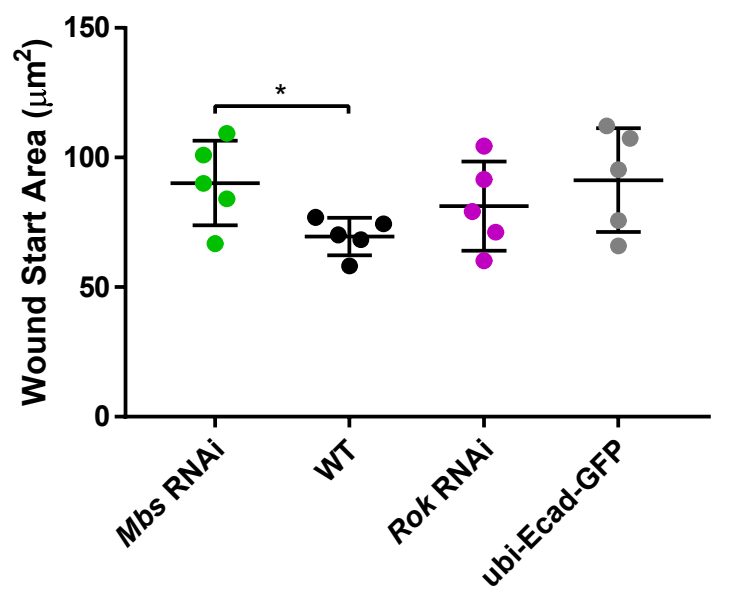

d

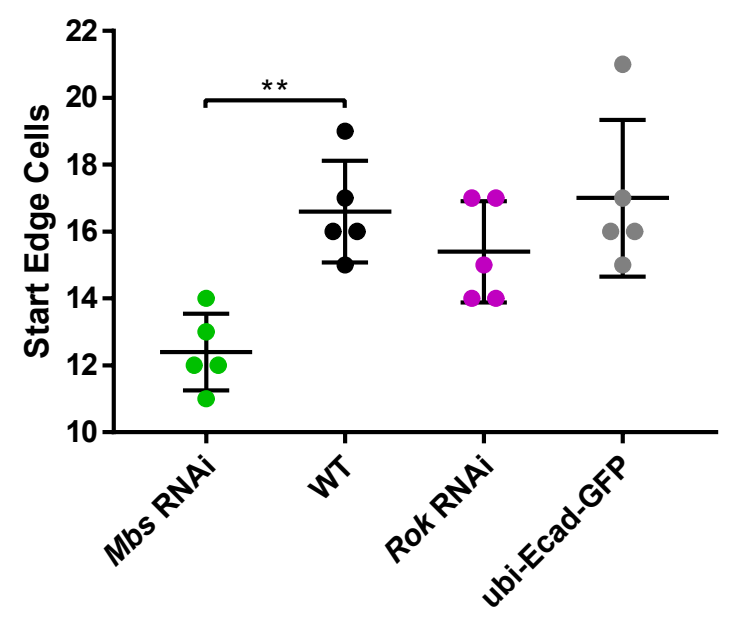

Supplementary Figure 6. Comparisons of features of Mbs RNAi, Rok RNAi, ubiEcad-GFP and WT wounds

a, Quantification of time of closure. Rok RNAi (unpaired $t$-test, $t=7.318, d f=8, p<0.0001$ ) and ubi-Ecad-GFP (unpaired $t$-test, $t=6.907, d f=8, p=0.0001$ ) wounds close in less time than WT wounds. Mbs RNAi wounds fail to close. $\mathbf{b}$, Quantification of wound start areas. WT wounds were smaller than Mbs RNAi wounds (unpaired $t$-test, $t=2.585, d f=8$, $p=0.0324)$. c, Quantification of number of cells killed. Fewer cells were killed in Mbs RNAi wounds than in WT (unpaired $t$-test, $t=4.906, d f=8, p=0.0012$ ). d, Quantification of starting wound edge cells. Mbs RNAi wounds had fewer starting edge cells than WT (unpaired $t$-test, $t=4.95, d f=8, p=0.0011$ ). a-d, Error bars $=\mathrm{SD}$. All statistical tests were performed against WT and were only reported if significant. 
a

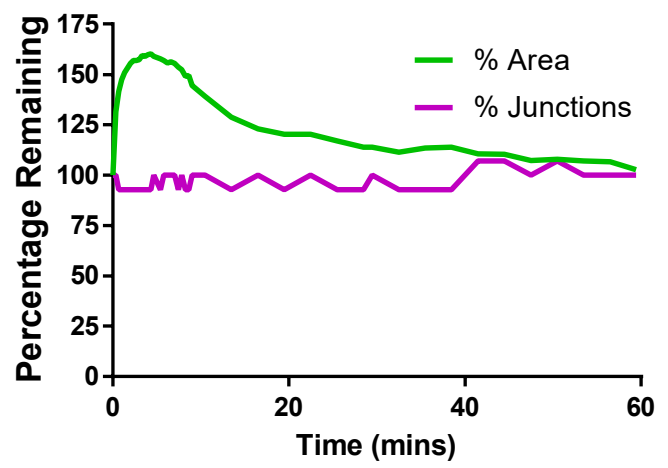

C

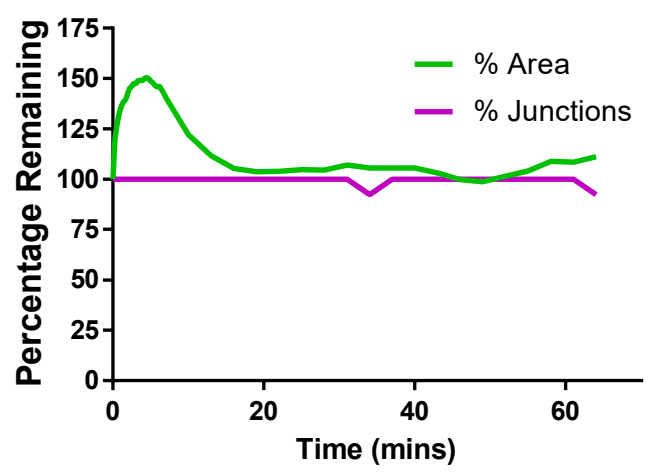

e

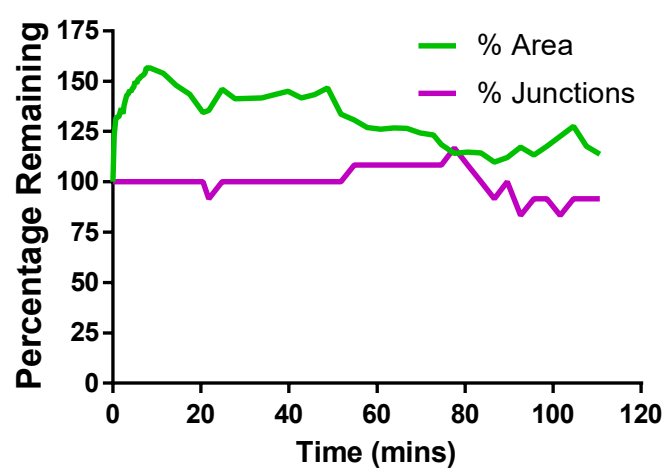

b

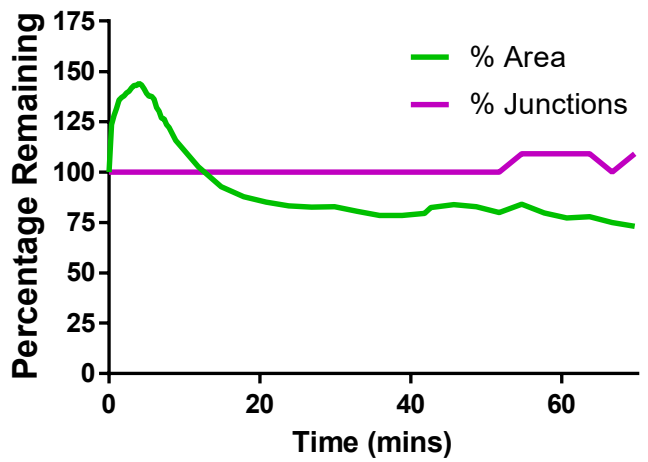

d

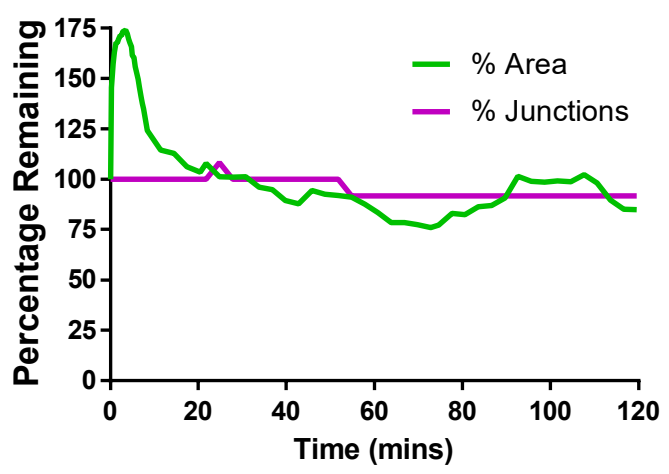

Supplementary Figure 7. Relationship between wound area changes and intercalation events in Mbs RNAi wing discs

a-e, Quantification of the percentage of starting wound edge junctions (magenta) and wound percentage area (green) for five Ecad-GFP/UAS-Mbs-RNAi; rn-GAL4/+ wing disc wounds. 
a

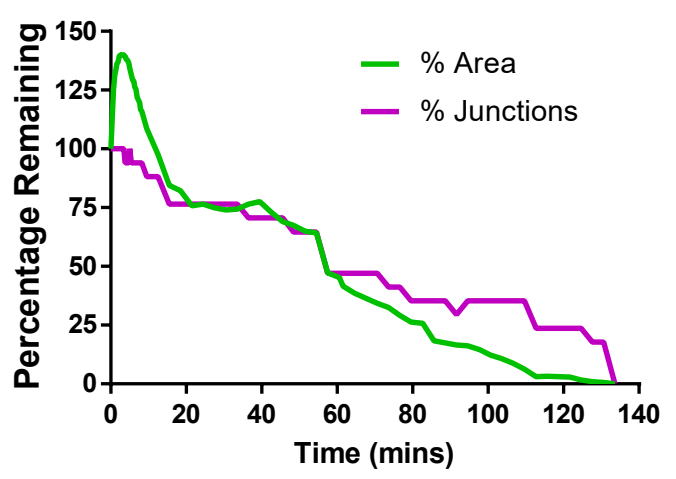

C

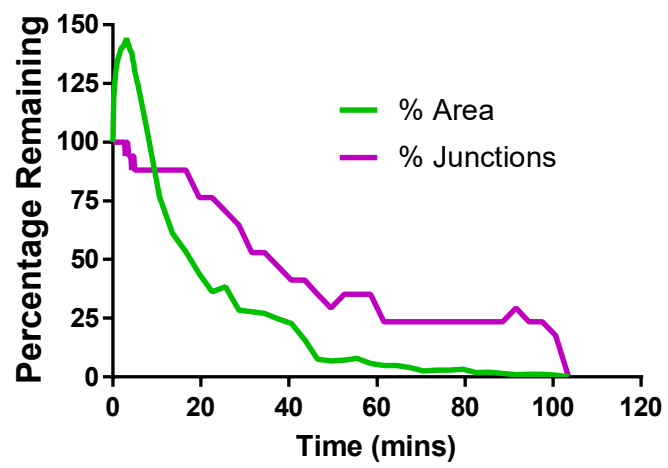

e

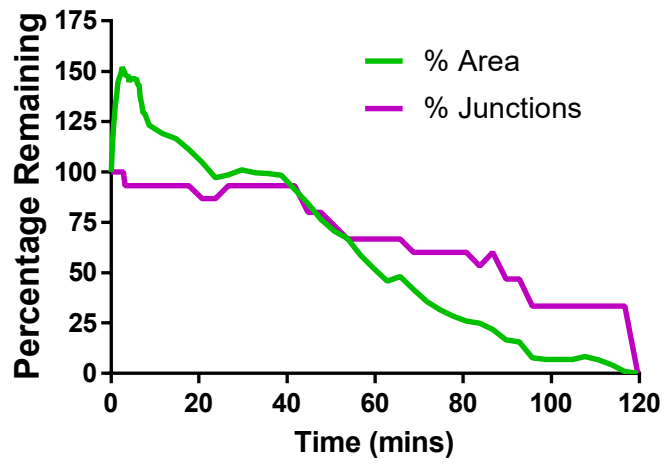

b

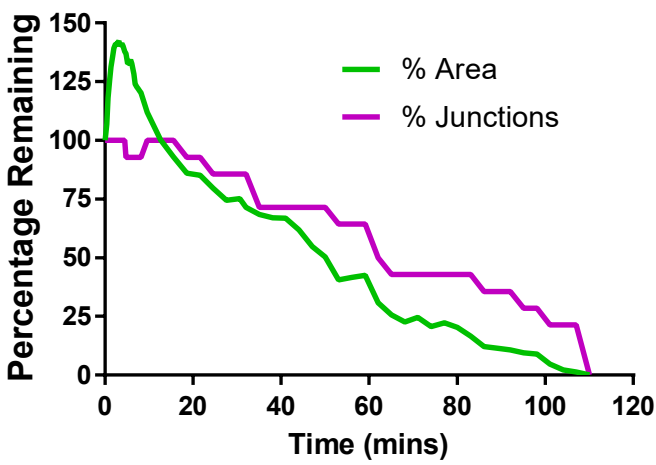

d

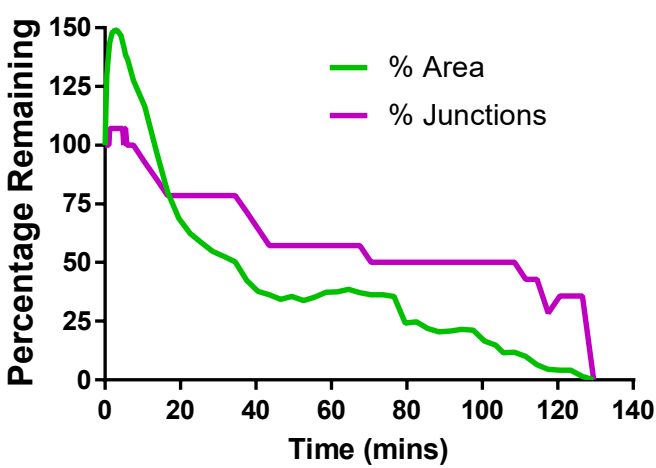

Supplementary Figure 8. Relationship between wound area changes and intercalation events in Rok RNAi wing discs

a-e, Quantification of the percentage of starting wound edge junctions (magenta) and wound percentage area (green) for five Ecad-GFP/UAS-Rok-RNAi; rn-GAL4/+ wing disc wounds. 
a

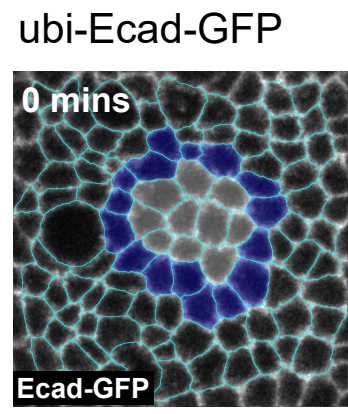

C

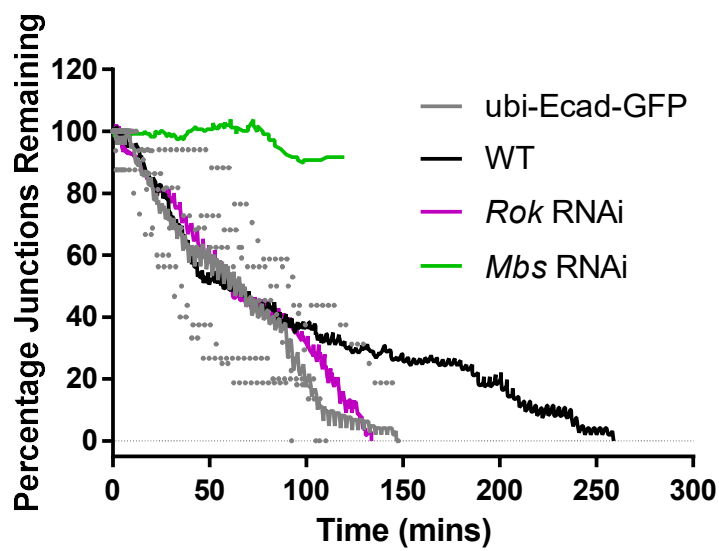

e

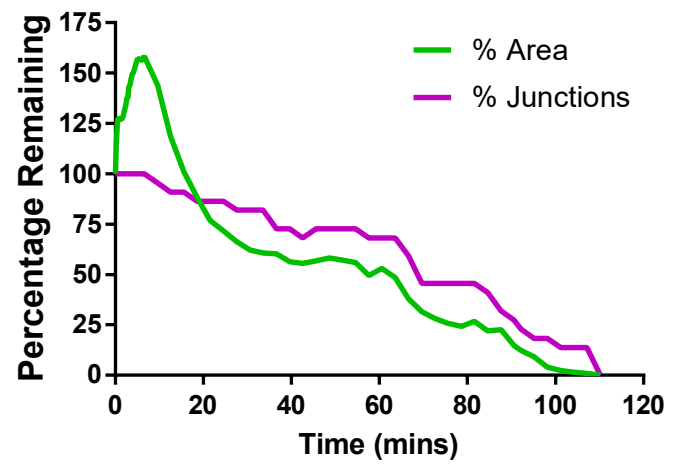

g

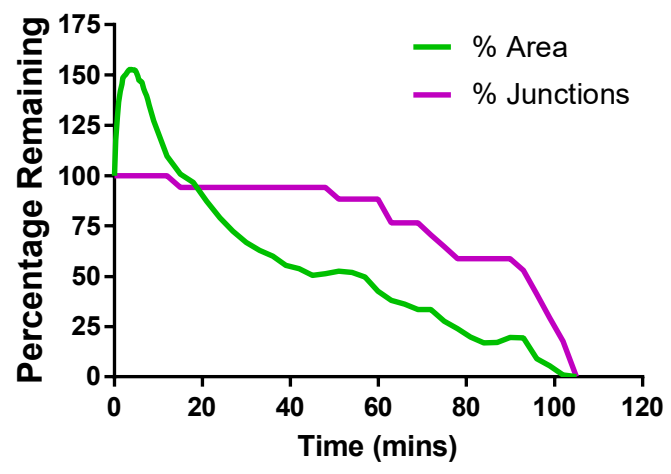

b
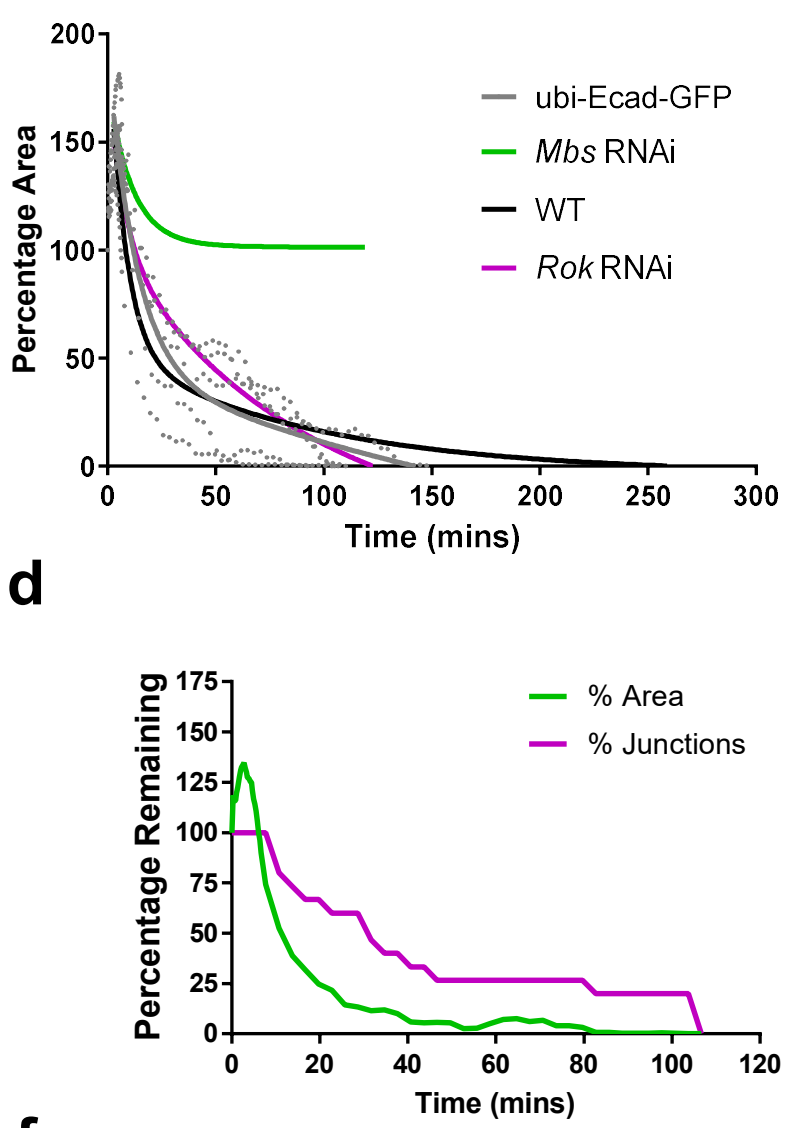

f

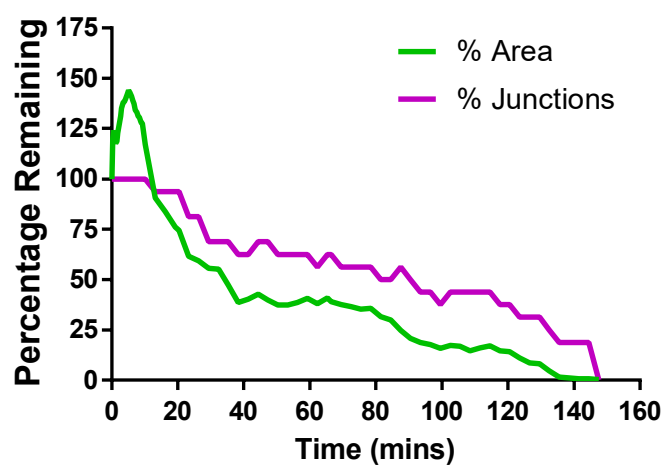

h

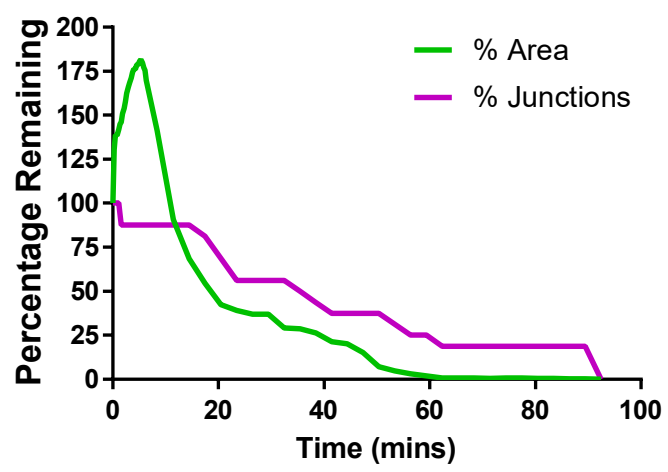




\section{Supplementary Figure 9. Wound closure dynamics in ubi-Ecad-GFP wing discs.}

a, Example of wound healing in a ubi-Ecad-GFP wing disc before wounding (left) and after wound closure (right). Starting wound edge cells are marked in dark blue. Images are adaptive projections of Ecad-GFP overlaid by skeletonised cell outlines in cyan (scale bar $=3 \mu \mathrm{m})$. b, Quantification of wound closure (as percentage of start wound area) over time in ubi-Ecad-GFP wing discs (grey, $n=5$ ) with a two-phase exponential decay fitted after 3 minutes. ubi-Ecad-GFP data is overlaid on two-phase exponential decays (from Fig. 5b) for WT (black), Rok RNAi (magenta) and Mbs RNAi (green). c, Quantification of the percentage of initial wound edge junctions over time for ubi-EcadGFP wing discs (colours and $n$ numbers as in $b$ ) with a moving average curve ( \pm 4 time points) shown. Data is overlaid on moving average curves (from Fig. 5e) for WT, Rok RNAi and Mbs RNAi. d-h, Quantification of the percentage of starting wound edge junctions (magenta) and wound percentage area (green) for five ubi-Ecad-GFP wing disc wounds. 
a Intercalation Disabled

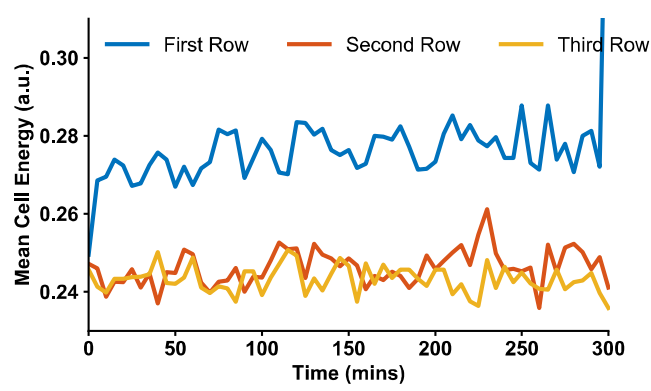

b Intercalation Enabled

C

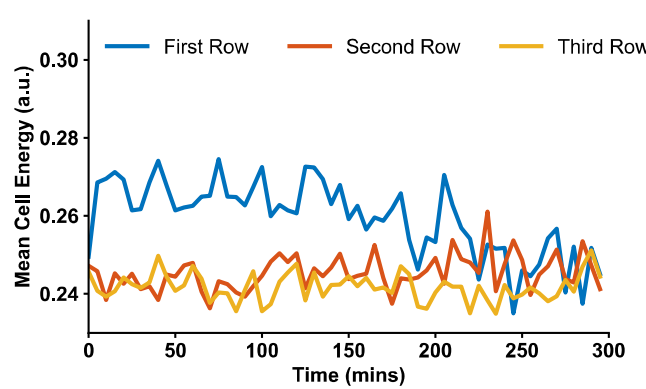

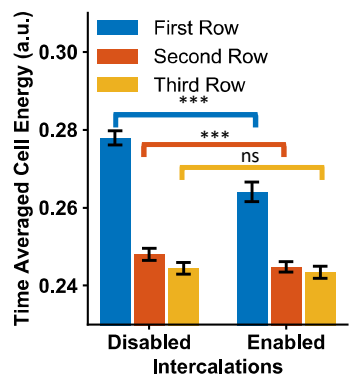

\section{Supplementary Figure 10. Effect of intercalations on cell energy}

a-b, Mean cell mechanical energy over time for the first three rows of cells with intercalations (a) enabled, and (b) disabled. c, Quantification of time averaged cell energy for the first three rows, with intercalations enabled and disabled, averaged over 12 simulations. With intercalations enabled, the mean cell area is lower in the first row (unpaired $t$-test, $t=14.8, d f=22, p<1 \mathrm{e}-12$ ), second row (unpaired $t$-test, $t=5.282, d f=22$, $p<1 \mathrm{e}-4$ ), and third row (unpaired $t$-test, $t=1.642, d f=22, p>1 \mathrm{e}-1$ ). Error bars $=\mathrm{SD}$.

\begin{tabular}{|c|c|c|c|c|c|c|}
\hline & Rok RNAi Tissue & Rok RNAi Wound & WT Tissue & WT Wound & Mbs RNAi Tissue & Mbs RNAi Wound \\
\hline Rok RNAi Tissue & & $<0.0001(0.8078)$ & $0.002(0.5845)$ & $<0.0001(0.95)$ & $<0.0001(0.913)$ & $<0.0001(0.8604)$ \\
\hline Rok RNAi Wound & & & $0.0167(0.5088)$ & $0.0019(0.5974)$ & $0.9368(0.1974)$ & $0.1516(0.3684)$ \\
\hline WT Tissue & & & & $0.0002(0.6889)$ & $0.0033(0.6667)$ & $0.0019(0.614)$ \\
\hline WT Wound & & & & & $0.0365(0.5167)$ & $0.2506(0.3263)$ \\
\hline Mbs RNAi Tissue & & & & & & $0.6884(0.2632)$ \\
\hline Mbs RNAi Wound & & & & & & \\
\hline
\end{tabular}

\section{Supplementary Table 1. Results of Kolmogorov Smirnov tests comparing vertex recoil rates in Rok RNAi, WT and Mbs RNAi wing discs}

Results of Kolmogorov Smirnov tests for pairwise comparisons of all combinations of vertex recoil rates at the wound edge and in the surrounding tissue in Rok RNAi, WT and Mbs RNAi wing discs. $p$ values and the Kolomogorov Smirnov D statistic (in brackets) are shown. Statistically significant comparisons are highlighted in green. 
Supplementary Video 1. Myosin II localisation during Drosophila wing disc wound closure. Time-lapse of the first hour after wounding in a $s q h^{A X 3}$; $s q h$-GFP, Ecad-tdTomato wing imaginal disc. Myosin II is marked by Sqh-GFP (magenta, maximum intensity projection) and cell outlines by Ecad-tdTomato (green, adaptive projection). Myosin II rapidly accumulates at the wound's edge in the manner of a purse string.

Supplementary Video 2. WT wound closure. Time-lapse of an Ecad-GFP/+; rnGAL4/+ (WT) wing disc from wounding to wound closure. An adaptive projection of Ecad-GFP (greyscale) is overlaid with skeletonised cell outlines (cyan). The wound itself (white) and initial wound edge cells (blue) are highlighted.

\section{Supplementary Video 3. Vertex model simulation of wound healing with} intercalations disabled. Red edges represent the wound edge and have increased line tension compared to the surrounding tissue (black edges). The wound fails to close during the simulation.

\section{Supplementary Video 4. Vertex model simulation of wound healing with} intercalations enabled. The same parameters are used as in Supplementary Video 3 , except that intercalations are enabled. The wound is able to close.

Supplementary Video 5. Analysing the first three rows of cells away from the wound. Time-lapse of an Ecad-GFP/+; rn-GAL4/+ (WT) wing disc from wounding to wound closure. An adaptive projection of Ecad-GFP (greyscale) is overlaid with skeletonised cell outlines (cyan). The wound itself is highlighted in white. The first (blue), second (orange) and third (yellow) rows of cells away from the wound are selected prior to wounding. These initial cell identities are propagated through time, regardless of whether the cell intercalates or not.

Supplementary Video 6. Wound closure in an Mbs RNAi wing disc. Time-lapse of an Ecad-GFP/UAS-Mbs-RNAi; $r n-G A L 4 /+$ wing disc after wounding. The wound fails to close during imaging. An adaptive projection of Ecad-GFP (greyscale) is overlaid with skeletonised cell outlines (cyan). The wound itself (white) and initial wound edge cells that do intercalate (blue) and do not intercalate (cyan) are highlighted.

Supplementary Video 7. Wound closure in a Rok RNAi wing disc. Time-lapse of an Ecad-GFP/UAS-Rok-RNAi; $r n-G A L 4 /+$ wing disc from wounding to wound closure. An adaptive projection of Ecad-GFP (greyscale) is overlaid with 
skeletonised cell outlines (cyan). The wound itself (white) and initial wound edge cells (blue) are highlighted. 